NISTIR 7887

\title{
Correction Factors for the NIST Free-Air Ionization Chambers Used to Realize Air Kerma from W-Anode X-Ray Beams
}

Stephen M. Seltzer

http://dx.doi.org/10.6028/NIST.IR.7887




NISTIR 7887

\section{Correction Factors for the NIST Free-Air Ionization Chambers Used to Realize Air Kerma from W-Anode X-Ray Beams}

Stephen M. Seltzer Radiation and Biomolecular Physics Division

Physical Measurement Laboratory

http://dx.doi.org/10.6028/NIST.IR.7887

October 2012

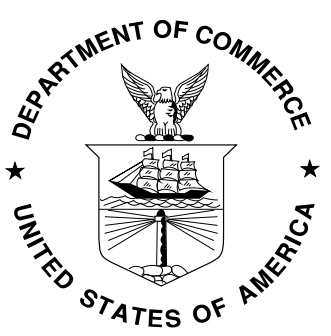

U.S. Department of Commerce Rebecca M. Blank, Acting Secretary

National Institute of Standards and Technology Patrick D. Gallagher, Under Secretary of Commerce for Standards and Technology and Director 


\title{
Correction Factors for the NIST Free-Air Ionization Chambers Used to Realize Air Kerma from W-Anode X-Ray Beams
}

\author{
October 2, 2012 \\ Stephen M. Seltzer \\ Guest Researcher \\ Dosimetry Group \\ Radiation and Biomolecular Physics Division \\ Physical Measurement Laboratory \\ National Institute of Standards and Technology \\ Gaithersburg, MD USA
}

\begin{abstract}
This report details analyses in the development of certain correction factors for free-air ionization chambers used by the National Institute of Standards and Technology to realize air kerma for its measurement standards and calibrations of W-anode X-ray beams. The correction factors dealt with in this report are the electron-loss correction, $k_{\mathrm{el}}$, the photon-scatter correction, $k_{\mathrm{sc}}$, the fluorescence-reabsorption correction, $k_{\mathrm{fl}}$, and the bremsstrahlung-reabsorption correction, $k_{\mathrm{br}}$.
\end{abstract}




\section{Introduction}

This report documents the development of certain correction factors for the parallel-plate free-air chambers (FACs) used by the National Institute of Standards and Technology (NIST) to realize air kerma for W-anode x-ray beams. These FACs are the Lamperti chamber for $\mathrm{x}$-ray beams of $10 \mathrm{kV}$ to $50 \mathrm{kV}$, the Ritz chamber for $\mathrm{x}$-ray beams of $20 \mathrm{kV}$ to $100 \mathrm{kV}$, and the Wyckoff-Attix chamber for x-ray beams of $50 \mathrm{kV}$ to $300 \mathrm{kV}$. Because this report restricts itself to new evaluations of particular FAC-geometrydependent correction factors, the reader is referred to previous publications (Lamperti et al., 1988; Lamperti and O’Brien, 2001) for more general descriptions of the FACs and the evaluation of the complete list of correction factors, including the historical methods some of which the present report replaces.

The quantity kerma is defined (ICRU, 2011) for ionizing uncharged particles as the quotient of $\mathrm{d} E_{\mathrm{tr}}$ by $\mathrm{d} m$, where $\mathrm{d} E_{\mathrm{tr}}$ is the mean sum of the initial kinetic energies of all the charged particles liberated in a mass $\mathrm{d} m$ of a material by the uncharged particles incident on $\mathrm{d} m$, thus

$$
K=\frac{\mathrm{d} E_{\mathrm{tr}}}{\mathrm{d} m}
$$

In the International System of Units (SI) (BIPM, 2006) kerma has units of $\mathrm{kg}^{-1}$; the special name for this unit is gray (Gy).

Technically, air kerma, $K_{\text {air }}$, is realized through a measurement of the related quantity exposure. Exposure, $X$, is the quotient of $\mathrm{d} q$ by $\mathrm{d} m$, where $\mathrm{d} q$ is the absolute value of the mean total charge of the ions of one sign produced when all the electrons and positrons liberated or created by photons incident on a mass $\mathrm{d} m$ of dry air are completely stopped in dry air, thus

$$
X=\frac{\mathrm{d} q}{\mathrm{~d} m}
$$

The SI unit of exposure is $\mathrm{C} \mathrm{kg}^{-1}$ (however, the older unit of Roentgen $(\mathrm{R}$ ) is still used by some, where $1 \mathrm{R}=2.58 \times 10^{-4} \mathrm{C} \mathrm{kg}^{-1}$ ). The ionization produced by electrons emitted in atomic/molecular relaxation processes is included in $\mathrm{d} q$. The ionization due to photons emitted by radiative processes (i.e., bremsstrahlung and fluorescence photons) is not to be included in dq. Except for this difference, significant at high energies, the exposure, as defined above, is the ionization analogue of the dry-air kerma.

The quantities exposure and air kerma can be related through use of the mean energy expended in a gas per ion pair formed, divided by the elementary charge, W/e, where $W$ is the mean energy expended in air per ion pair formed when the initial kinetic 
energy of a charged particle is completely dissipated in the air, and e is the elementary charge. Then

$$
K_{\mathrm{air}} \approx X \cdot(W / \mathrm{e}) /(1-\bar{g})
$$

The quantity $g$ is the fraction of the kinetic energy of electrons (and positrons) liberated by the photons that is lost in radiative processes (mainly bremsstrahlung) in air. In Eq. (3), $\bar{g}$ is the mean value of $g$ averaged over the distribution of the air kerma with respect to electron energy.

A schematic of a generic parallel-plate FAC is shown in Fig. 1. The FAC is a shielded container open to the atmosphere into which a portion of the x-ray beam enters through a defining aperture, passes between parallel collecting and high-voltage plates, and leaves through an exit aperture. The dimensions of the components are designed to allow complete slowing down of all electrons produced, which are collected by the electric field across the collecting volume.

The results of a FAC measurement for x-ray beams are then analyzed according to the measurement equation

$$
K_{\text {air }} \approx(W / \mathrm{e}) \frac{Q_{\text {net }}}{\rho_{\text {air }} V_{\text {eff }}(1-\bar{g})} \prod_{i} k_{i},
$$

where $Q_{\text {net }}$ is the measured net ion current (corrected for cosmic-ray and systemgenerated background), $\rho_{\text {air }}$ is the density of air, and $V_{\text {eff }}$ is the product of the aperture area and the length of the collecting volume. Equation (4) is an elaboration of combining Eqs. (2) and (3) in which the mass has been replaced by the product $\rho_{\text {air }} V_{\text {eff }}$ and the application of the various necessary corrections is shown. The radiative-loss correction $\bar{g}$ is very small, effectively zero, for these x-ray beams, and $k_{i}$ are correction factors for air attenuation, photon scatter, electron loss, etc., within the FAC. It is assumed that the correction factors include also those for humidity, ion recombination, and ambient pressure and temperature (to correct to reference conditions), but these are not the subject of this report (the correction for humidity was discussed in Seltzer et al. (2003), in which the value of 0.998 used by the NIST was verified).

Before continuing, it should be noted that the approximate equality in Eqs. (3) and (4) is used here to reflect the fact that exposure includes the charge of electrons or ions liberated by the incident photons whereas $W$ pertains only to the charge produced during the slowing down of these electrons. Thus the initial charge created by the interaction of the photons should be discounted when transferring the exposure measurement for the determination of air kerma. This difference, although relatively small, tends to become more significant as the photon energy decreases. Additionally, $W$ is not constant as perhaps implied in Eqs. (3) and (4), but is known to increase at low energies (ICRU, 1979). At energies for which the variation of $W$ with energy becomes important, one 
should consider also the effect of this increase. The initial-ion correction has been considered by Büermann et al. (2006), and by Takata and Begum (2008). The NIST does not yet include this correction; it appears to be relatively small, i.e., rather close to unity, but does seem to become more significant, approaching about 0.995 for standard x-ray beams with the lowest mean energies.

\section{Correction Factors}

The correction factors of concern in this report are mainly those for electron loss, photon scatter, fluorescence reabsorption, and bremsstrahlung reabsorption. The first two of these have been evaluated and used for more than 50 years (see Lamperti et al., 1988; Lamperti and O’Brien, 2001; and references therein), but the advent of reliable radiationtransport Monte Carlo calculations has allowed more accurate and consistent data, including that for the latter two correction factors, which although small had not been considered until about a decade ago.

Electron-loss correction, $k_{\mathrm{el}}$. Energetic electrons can leave (and enter) the collection volume, with only a portion of their energy expended in ionization being collected. The collecting volume is defined by the area of the collecting plate (collector length $x$ collector width in Fig. 1) and the electrode separation.

Photon-scatter correction, $k_{\mathrm{sc}}$. Ionization produced by electrons resulting from photons scattered out of the aperture-defined beam is not included in the definitions of exposure and of air kerma.

Fluorescence-reabsorption correction, $k_{\mathrm{fl}}$. The ionization collected due to reabsorption of fluorescence photons is not included in the definitions of exposure and of air kerma.

Bremsstrahlung-reabsorption correction, $k_{\mathrm{br}}$. The ionization collected due to reabsorption of bremsstrahlung photons is not included in the definitions of exposure and of air kerma. This is associated with but separate from the $(1-g)$ correction. The NIST currently assumes $\bar{g}$ in Eq. (4) is identically zero for its calibration x-ray beams. If the appropriate small values were to be used, then the use of $k_{\text {br }}$ would correct for effects of reabsorption. Although the following analyses will carry along results for $k_{\mathrm{br}}$, the final recommendation will be an estimated uncertainty associated with the assumption that $k_{\mathrm{br}} /(1-\bar{g})=1$.

\section{Data for $\boldsymbol{k}_{\mathrm{el}}, \boldsymbol{k}_{\mathrm{sc}}, \boldsymbol{k}_{\mathrm{fl}}$, and $\boldsymbol{k}_{\mathrm{br}}$ for Monoenergetic Photons}

The source of data for the NIST correction factors is the Monte Carlo calculations reported by Burns (2001) for the FACs of NIST and other national metrology institutes. These calculations were a follow-on to earlier work (Burns, 1999), but using a Version VI of the EGSnrc code that included the transport of fluorescence $\mathrm{x}$ rays and of Auger 
and Coster-Kronig electrons. The critical dimensions used for the NIST FACs are listed in Table 1. In addition to these dimensions, Burns used a gap between electrodes and side walls of $2 \mathrm{~cm}$ for the Lamperti and Ritz chambers, and of $5 \mathrm{~cm}$ for the WyckoffAttix chamber. He assumed electrodes and wall materials to be aluminum and an electrode thickness of $1 \mathrm{~cm}$. The composition of air was taken as that listed in ICRU Report 37 (ICRU, 1984), and the histories of photons and electrons were terminated at a kinetic-energy cut-off of $1 \mathrm{keV}$. The number of histories was such that Burns states that the relative statistical standard uncertainty of all results is less than $0.01 \%$.

Burns (1999) performed some sensitivity studies to assess uncertainties arising from his model of the chambers. He estimates the relative standard uncertainties to be $0.05 \%$ for $k_{\mathrm{el}}$ and $0.03 \%$ for $k_{\mathrm{sc}}\left(k_{\mathrm{fl}}\right.$ and $k_{\mathrm{br}}$ were not considered in this earlier study). In a later report, Burns (2003) used the Monte Carlo code PENELOPE to re-evaluate the correction factors for the BIPM FACs. From comparisons among the various results, he estimates a relative uncertainty of $0.03 \%$ for $k_{\mathrm{sc}}$ and from $0.03 \%$ to $0.05 \%$ for $k_{\mathrm{fl}}$, depending on the chamber size. Moreover, now with results from different methods of electron transport, he then estimates a relative uncertainty in $k_{\mathrm{el}}$ of from $0.01 \%$ to 0.09 $\%$, depending on the x-ray beam energy and thus the chamber used. These latter results are difficult to transfer to the NIST chambers, as the dimensions differ somewhat. However, it seems reasonable to assign to the NIST chambers the relative standard uncertainties due to the computational models employed to be those given in Table 2 .

The data supplied by Burns (private communication, 2001) were in the form of tables for each of the NIST FACs, and are reproduced in Table 3. These monoenergetic data were, however, processed by Burns from his Monte Carlo scores in terms of a concatenation of separate classes of energy deposition whose ratios yield the desired quantities. Using his notation and the quantities listed in Table 3, we can recover the essential scores, each as a function of photon energy:

$$
\begin{aligned}
& P_{\text {dep }}=\frac{(1-g) P_{\text {rel }}}{k_{\text {eq }}}, \\
& P_{\text {col }}=\frac{(1-g) P_{\text {rel }}}{k_{\mathrm{eq}} k_{\mathrm{el}}}, \\
& S_{\text {col }}=\left(\frac{1}{k_{\mathrm{sc}}}-1\right) \frac{(1-g) P_{\mathrm{rel}}}{k_{\mathrm{eq}} k_{\mathrm{el}}}, \\
& F_{\mathrm{col}}=\left(\frac{1}{k_{\mathrm{fl}}}-1\right) \frac{(1-g) P_{\mathrm{rel}}}{k_{\mathrm{eq}} k_{\mathrm{el}} k_{\mathrm{sc}}}, \\
& G_{\mathrm{col}}=\left(\frac{1}{k_{\mathrm{br}}}-1\right) \frac{(1-g) P_{\mathrm{rel}}}{k_{\mathrm{eq}} k_{\mathrm{el}} k_{\mathrm{sc}} k_{\mathrm{fl}}} .
\end{aligned}
$$


The normalization of the quantities given in Eqs. (5) (governed by that of $P_{\text {rel }}$ ) is unimportant, as we are ultimately interested only in their ratios. Indeed, we see then that the monoenergetic results are simply:

$$
\begin{aligned}
k_{\mathrm{el}}(E) & =\frac{P_{\mathrm{dep}}(E)}{P_{\mathrm{col}}(E)}, \\
k_{\mathrm{sc}}(E) & =\frac{P_{\mathrm{col}}(E)}{P_{\mathrm{col}}(E)+S_{\mathrm{col}}(E)}, \\
k_{\mathrm{fl}}(E) & =\frac{P_{\mathrm{col}}(E)+S_{\mathrm{col}}(E)}{P_{\mathrm{col}}(E)+S_{\mathrm{col}}(E)+F_{\mathrm{col}}(E)}, \\
k_{\mathrm{br}}(E) & =\frac{P_{\mathrm{col}}(E)+S_{\mathrm{col}}(E)+F_{\mathrm{col}}(E)}{P_{\mathrm{col}}(E)+S_{\mathrm{col}}(E)+F_{\mathrm{col}}(E)+G_{\mathrm{col}}(E)} .
\end{aligned}
$$

For a photon spectrum, however, it is formally required to integrate over the numerators and denominators of Eqs. (6). In order to facilitate interpolation for such integration, it was decided to fit and thus smooth the quantities on the right-hand side of Eqs. (6). It was noticed that some of the quantities reconstructed from Eqs. (5) displayed inconsistent results at the lowest energy or energies (possibly due to energy cut-offs in the Monte Carlo calculations); in particular, there should be no contribution by fluorescence re-absorption below the K-edge energy of $3.206 \mathrm{keV}$ for Ar. Therefore in some cases the fitted data were extrapolated down to these lower energies to insure smoothness. Issues of accuracy for these lowest energies are unimportant because x-ray spectra of NIST calibration beams rarely extend down to these energies and, in any case, the relative energy fluence would be quite small.

The original and adopted smoothed data are given in Tables 4 and 5, respectively. Reconstructed results for the monoenergetic-photon correction factors from the smoothed data via Eq. (6) are given in Table 6. These are compared with the original Burns data in Figs. 2 - 4. The modified data are not necessarily better, but they do follow the original rather closely and are indeed smoother. Table 7 gives estimated relative uncertainties due to the smoothing process, calculated as the relative root-mean-square deviation between the data of Tables 3 and 6 . These are combined (in quadrature) with the results that were given in Table 2 for the estimated total relative standard uncertainties inherent in the basic data adopted for calculating correction factors for the NIST FACs, and these are listed in Table 8. That is, it is assumed that any additional uncertainty is to be contributed by that in the input spectra for the incident $\mathrm{x}$-ray beam.

As regards $k_{\mathrm{br}}$, one can form the product $k_{\mathrm{br}} /(1-g)$ from Burns' Monte Carlo results in Table 3, and investigate its difference from unity. Such results suggest an estimate of the relative standard uncertainties associated with the assumption that $\mathrm{kbr}_{\mathrm{br}} /(1-\bar{g})=1$ of $0.02 \%$ for the Lamperti chamber, $0.02 \%$ for the Ritz chamber, and $0.03 \%$ for the Wyckoff-Attix chamber. These have been added to Table 8 for completeness. 


\section{Results for the NIST Standard Beam Qualities}

There has developed a fair amount of information on the $\mathrm{W}$-anode $\mathrm{x}$-ray fluence spectra that the NIST uses in its measurement standards and calibration services (see, Seelentag et al., 1979; Eisenhower et al., 1983; Iles, 1987; Peaple et al., 1989; Laitano et al., 1989; 1991; Ankerhold, 2000), as well as the tools to estimate such fluence spectra for a wide range of conditions (see, e.g., Cranley et al., 1997). C.G. Soares of the NIST collected much of this information in digital form and made it available (private communication, 1994). These spectra, including the NIST traditional and ISO beams (ISO, 1996), number some 200 versions from independent measurements (from the institutions GSF, Harwell, ENEA, PTB, and NIST) and calculations (from the NRPB), thus often providing multiple versions (up to 5) for many of the NIST beam qualities.

The smoothed data in Table 5 were integrated over these available fluence spectra, the ratios of the integrated values were taken as indicated in Eq. (6), and the results from multiple versions of the spectra were averaged. The adopted results are listed in Table 9 for the traditional NIST beams qualities, in Table 10 for the ISO beam qualities, and in Table 11 for the BIPM/CCRI beam qualities used for international comparisons. There were no fluence spectra available for the more recently added NIST beam qualities M80 and M120, so the correction factors were obtained by interpolation as a function of the half-value layer for the Ritz and for the Wyckoff FACs.

The variations in results from multiple spectra for the same beam quality suggest that a reasonable estimate of the relative standard uncertainty of the results is $20 \%$ of the (absolute) difference of the correction factor from unity. Because the correction factors are so close to unity, this estimated relative standard uncertainties of the correction factors themselves due to assumed fluence spectrum is never more than about $0.01 \%$. This then suggests that the uncertainties listed in Table 8 are appropriate for the final results in Tables 9 and 10.

\section{Final Remarks}

This work is the culmination of efforts started in 2001 to produce correction factors for the NIST FACs. The results produced in various analyses over the last decade are rather consistent, but the present results should be considered as the most complete, with a perhaps more careful uncertainty analysis. The analyses have also included the correction for attenuation within the FAC, $k_{\mathrm{at}}$, using standard reference data for the photon attenuation coefficients, presumably consistent with the Monte Carlo calculations by Burns. Because of ambiguity on the role of coherent scattering in the analytical calculations of the attenuation, and on the uncertainty of the underlying photoninteraction cross sections, the calculated $k_{\mathrm{at}}$ values were intended only to confirm measured values, which could be readily obtained (although not without some difficulty). Thus these values are not presented here, although agreement with measured values is reasonably good. 


\section{Acknowledgements}

This work was conducted over the years in close collaboration with Michelle O'Brien, the NIST expert for air-kerma measurement standards for X-ray beams and ultimate user of these results. Of course, this work would not have been possible without the Monte Carlo results and background explanations supplied by David Burns of the BIPM, nor without the collection of x-ray-beam fluence spectra provided by Christopher Soares of the NIST. The efforts of the author were partially supported through contract SB1341-11-RQ-0398 with the Dosimetry Group, Radiation and Biomolecular Physics Division, National Institute of Standards and Technology. 


\section{References}

Ankerhold, A. (2000). Catalogue of X-Ray Spectra and their Characteristic Data - ISO and DIN Radiation Qualitites, Therapy and Diagnostic Radiation Qualities, Unfiltered X-Ray Spectra, PTB Report PTB-Dos-34, Physikalisch-Technische Bundesanstalt, Braunschweig, Germany.

Büermann, I., Grosswendt, B.,. Kramer, H.-M., Selbach, H.-J., Gerlach, M., Hoffmann, M., and Krumrey, M. (2006). "Measurement of the x-ray mass energy-absorption coefficient using $3 \mathrm{keV}$ to $10 \mathrm{keV}$ synchrotrton radiation,” Phys. Med. Biol. 51, 5125-5150.

BIPM (2006). Bureau International des Poids et Mesures, Le Systéme International d'Unités (SI), The Initernational System of Units (SI), 8th edition (Bureau International des Poids et Mesures, Sèvres).

Burns, D.T. (1999). "Consistent set of calculated values for electron-loss and photonscatter corrections for parallel-plate free-air chambers,” CCRI(I)/99-4, BIPM, Sèvres, France.

Burns, D.T. (2001). "Free-air chamber correction factors for electron loss, photon scatter, fluorescence and bremsstrahlung,” CCRI(I)/01-36, BIPM, Sèvres, France.

Cranley,K., Gilmore, BJ., Fogarty, G.W.A., and Desponds, L. (1997). Catalogue of Diagnostic X-ray Spectra and Other Data, Report No. 78, Institute of Physics and Engineering in Medicine, York, UK.

Eisenhower, E.H., Ehrlich, M., Soares, C., Schima, F.J., and Seltzer, S.M. (1983). Quality Assurance for Measurements of Ionizing Radiation (Annual Report for FY 1982), USNRC Report NUREG/CR-3120, Washington, D.C.

ICRU (1979). International Commission on Radiation Units and Measurements, Average Energy Required to Produce an Ion Pair, ICRU Report 31 (International Commission on Radiation Units and Measurements, Bethesda, MD).

ICRU (2011). International Commission on Radiation Units and Measurements, Fundamental Quantities and Units for Ionizing Radiation, ICRU Report 85a (International Commission on Radiation Units and Measurements, Bethesda, MD).

Iles, W.J. (1987). The Computation of Bremsstrahlung X-Ray Spectra over an Energy Range $15 \mathrm{keV}$ to $300 \mathrm{keV}$. Document NRPB-R204, National Radiological Protection Board, Chilton, UK. 
ISO (1996). International Organization for Standardization, $X$ and gamma reference radiations for calibrating dosimeters and dose rate meters and for determining their responses as a function of photon energy -- Part 1.: Radiation characteristics and production methods, ISO/IS 4037-1:1996 (International Organization for Standardization, Geneva).

Laitano, R.F. Pani, R. Pellegrini, R., and Toni, M.P. (1989). Energy Distributions and Air Kerma Rates of ISO and BIPM Reference Filtered X-Radiations, Technical Report ENEA RT/PAS(89), ENEA (National Agency for Atomic Energy), Rome, Italy.

Laitano, R.F. Pani, R. and Pellegrini, R.(1991). "Determination od x-ray spectra and of the scattered component up to $300 \mathrm{kV}$," Med. Phys. 18, 934-938.

Lamperti, P.J., Loftus, T.P., and Loevinger, R. (1988). NBS Measurement Services: Calibration of X-Ray and Gamma-Ray Measuring Instruments, National Bureau of Standards Special Publication 250-16, Gaithersburg, MD.

Lamperti, P.J., and O'Brien, M. (2001). NIST Measurement Services: Calibration of XRay and Gamma-Ray Measuring Instruments, National Institute of Standards and Technology Special Publication 250-58, Gaithersburg, MD.

Peaple, L.H.J., Birch, R., and Marshall, M. (1989). Measurement of the ISO Series of Filtered Radiations, Report AERE-R-13424, Atomic Energy Research Establishment, Harwell, England.

Seelentag, W.W., Panzer, W., Drexler, G., Platz, L., and Santner, F. (1979). A Catalogue of Spectra for the Calibration of Dosemeters, GSF Bericht 560. Gesellschaft fur Strahlen und Umweltforschung MBH, Munchen, Germany.

Seltzer, S.M., Lamperti, P.J., Loevinger, R., Mitch, M.G., Weaver, J.T., and Coursey, B.M. (2003). "New National Air-Kerma-Strength Standards for ${ }^{125} \mathrm{I}$ and ${ }^{103} \mathrm{Pd}$ Brachytherapy Seeds," NIST J. Res. 108, 337-358.

Takata, N., and Begum, A. (2008). "Corrections to air kerma and exposure measured with free air ionization chambers for charge of photoelectrons, Compton electrons and Auger electrons," Radiat. Prot. Dosim. 130, 410-418. 
Table 1. Critical dimensions for the NIST parallel-plate free-air chambers. All dimensions are in units of $\mathrm{cm}$.

\begin{tabular}{|l|c|c|c|}
\hline & $\begin{array}{c}\text { Lamperti } \\
(10 \mathrm{kV}-50 \mathrm{kV})\end{array}$ & $\begin{array}{c}\text { Ritz } \\
(20 \mathrm{kV}-100 \mathrm{kV})\end{array}$ & $\begin{array}{c}\text { Wyckoff-Attix } \\
(50 \mathrm{kV}-300 \mathrm{kV})\end{array}$ \\
\hline Aperture diameter & 0.5 & 1.0 & 1.0 \\
\hline Collector length & 1.0 & 7.0 & 10.1 \\
\hline Collector width & 5.0 & 9.0 & 27.0 \\
\hline Electrode separation & 4.0 & 9.0 & 27.0 \\
\hline Attenuation length & 3.9 & 12.7 & 30.8 \\
\hline
\end{tabular}

Table 2. Estimated relative standard uncertainties due to computational models adopted for the NIST FACs.

\begin{tabular}{|c|c|c|c|}
\hline $\begin{array}{c}\text { Correction } \\
\text { Factor }\end{array}$ & $\begin{array}{c}\text { Lamperti } \\
(10 \mathrm{kV}-50 \mathrm{kV})\end{array}$ & $\begin{array}{c}\text { Ritz } \\
(20 \mathrm{kV}-100 \mathrm{kV})\end{array}$ & $\begin{array}{c}\text { Wyckoff-Attix } \\
(50 \mathrm{kV}-300 \mathrm{kV})\end{array}$ \\
\hline$k_{\mathrm{el}}$ & $0.05 \%$ & $0.05 \%$ & $0.09 \%$ \\
\hline$k_{\mathrm{sc}}$ & $0.03 \%$ & $0.03 \%$ & $0.03 \%$ \\
\hline$k_{\mathrm{fl}}$ & $0.05 \%$ & $0.05 \%$ & $0.03 \%$ \\
\hline
\end{tabular}


Table 3. Original data from Burns' Monte Carlo calculations for NIST FACs. Note that values of $k_{\mathrm{el}}$ shown as 1.0 indicate that Burns evidently imposed this lower limit rather than giving the results from the ratio shown in Eq. (6). The various quantities are indicated in the text. The large number of significant figures is to preserve the data as transmitted.

a. Lamperti chamber.

\begin{tabular}{|c|c|c|c|c|c|c|c|}
\hline$E / \mathrm{keV}$ & $(1-g)^{-1}$ & $k_{\mathrm{eq}}$ & $k_{\mathrm{el}}$ & $k_{\mathrm{sc}}$ & $k_{\mathrm{fl}}$ & $k_{\mathrm{br}}$ & $P_{\text {rel }}$ \\
\hline 2 & 1.0000000 & 0.9997988 & 1.0 & 0.9980880 & 1.0000000 & 1.0000000 & $4.969 \mathrm{E}-19$ \\
\hline 4 & 1.0000028 & 0.9999972 & 1.0 & 0.9969588 & 0.9942037 & 0.9999959 & $8.651 \mathrm{E}-18$ \\
\hline 6 & 1.0000010 & 0.9997953 & 1.0 & 0.9976122 & 0.9956900 & 1.0000000 & $4.908 \mathrm{E}-18$ \\
\hline 8 & 1.0000120 & 0.9993245 & 1.0 & 0.9980929 & 0.9964462 & 0.9999954 & $3.012 \mathrm{E}-18$ \\
\hline 10 & 1.0000282 & 1.0005597 & 1.0 & 0.9982881 & 0.9970240 & 0.9999870 & $1.702 \mathrm{E}-18$ \\
\hline 12 & 1.0000557 & 0.9991425 & 1.0 & 0.9986930 & 0.9973521 & 0.9999813 & $1.246 \mathrm{E}-18$ \\
\hline 14 & 1.0000841 & 0.9999159 & 1.0 & 0.9985291 & 0.9975836 & 0.9999881 & $9.803 \mathrm{E}-19$ \\
\hline 16 & 1.0000991 & 0.9986722 & 1.0 & 0.9986802 & 0.9979104 & 0.9999662 & $6.502 \mathrm{E}-19$ \\
\hline 18 & 1.0001212 & 1.0010093 & 1.0 & 0.9988290 & 0.9980633 & 0.9999629 & $5.313 \mathrm{E}-19$ \\
\hline 20 & 1.0001339 & 1.0049185 & 1.0 & 0.9989213 & 0.9982463 & 0.9999654 & $3.978 \mathrm{E}-19$ \\
\hline 22 & 1.0001722 & 0.9986311 & 1.0 & 0.9989377 & 0.9984463 & 0.9999728 & $3.338 \mathrm{E}-19$ \\
\hline 24 & 1.0001486 & 0.9998514 & 1.0 & 0.9990345 & 0.9985611 & 0.9999669 & $2.889 \mathrm{E}-19$ \\
\hline 26 & 1.0001149 & 0.9975016 & 1.0 & 0.9989899 & 0.9986761 & 0.9999650 & $2.511 \mathrm{E}-19$ \\
\hline 28 & 1.0001800 & 0.9975803 & 1.0 & 0.9988816 & 0.9987150 & 0.9999731 & $2.227 \mathrm{E}-19$ \\
\hline 30 & 1.0002288 & 0.9980142 & 1.0 & 0.9987269 & 0.9988919 & 0.9999514 & $1.704 \mathrm{E}-19$ \\
\hline 32 & 1.0002138 & 1.0010957 & 1.0 & 0.9990631 & 0.9988387 & 0.9999615 & $1.529 \mathrm{E}-19$ \\
\hline 34 & 1.0002050 & 1.0002259 & 1.0002797 & 0.9990535 & 0.9990175 & 0.9999753 & $1.408 \mathrm{E}-19$ \\
\hline 36 & 1.0002150 & 1.0043660 & 1.0016750 & 0.9989713 & 0.9990364 & 0.9999441 & $1.285 \mathrm{E}-19$ \\
\hline 38 & 1.0003100 & 1.0024512 & 1.0056470 & 0.9990027 & 0.9991120 & 0.9999691 & $1.197 \mathrm{E}-19$ \\
\hline 40 & 1.0001991 & 1.0002266 & 1.0135405 & 0.9987202 & 0.9991943 & 0.9999642 & $1.016 \mathrm{E}-19$ \\
\hline 42 & 1.0002144 & 0.9989974 & 1.0267239 & 0.9991060 & 0.9992517 & 0.9999659 & $9.620 \mathrm{E}-20$ \\
\hline 44 & 1.0003089 & 1.0018165 & 1.0445255 & 0.9990351 & 0.9992840 & 0.9999639 & $9.136 \mathrm{E}-20$ \\
\hline 46 & 1.0002494 & 0.9993876 & 1.0702046 & 0.9989927 & 0.9993256 & 0.9999588 & $8.719 \mathrm{E}-20$ \\
\hline 48 & 1.0002166 & 0.9935509 & 1.0982407 & 0.9990157 & 0.9992944 & 0.9999566 & $8.383 \mathrm{E}-20$ \\
\hline 50 & 1.0002638 & 0.9995694 & 1.1251879 & 0.9988022 & 0.9993390 & 0.9999602 & $7.821 \mathrm{E}-20$ \\
\hline
\end{tabular}


b. Ritz chamber.

\begin{tabular}{|c|c|c|c|c|c|c|c|}
\hline$E / \mathrm{keV}$ & $(1-g)^{-1}$ & $k_{\mathrm{eq}}$ & $k_{\mathrm{el}}$ & $k_{\mathrm{sc}}$ & $k_{\mathrm{fl}}$ & $k_{\mathrm{br}}$ & $P_{\mathrm{rel}}$ \\
\hline 2 & 1.0000000 & 1.0000000 & 1.0 & 1.0000000 & 1.0000000 & 1.0000000 & $1.156 \mathrm{E}-21$ \\
\hline 4 & 1.0000002 & 0.9998972 & 1.0 & 0.9938854 & 0.9912199 & 0.9999996 & $9.745 \mathrm{E}-17$ \\
\hline 6 & 1.0000000 & 1.0000000 & 1.0 & 0.9947642 & 0.9934328 & 1.0000000 & $1.052 \mathrm{E}-16$ \\
\hline 8 & 1.0000017 & 0.9997311 & 1.0 & 0.9953721 & 0.9945537 & 0.9999994 & $7.485 \mathrm{E}-17$ \\
\hline 10 & 1.0000300 & 0.9999700 & 1.0 & 0.9962687 & 0.9953324 & 0.9999838 & $4.523 \mathrm{E}-17$ \\
\hline 12 & 1.0000530 & 0.9999470 & 1.0 & 0.9966030 & 0.9959413 & 0.9999818 & $3.365 \mathrm{E}-17$ \\
\hline 14 & 1.0000669 & 0.9999331 & 1.0 & 0.9966397 & 0.9965215 & 0.9999747 & $2.653 \mathrm{E}-17$ \\
\hline 16 & 1.0000837 & 0.9999163 & 1.0 & 0.9973156 & 0.9968536 & 0.9999656 & $1.790 \mathrm{E}-17$ \\
\hline 18 & 1.0001129 & 0.9998871 & 1.0 & 0.9972494 & 0.9970578 & 0.9999562 & $1.464 \mathrm{E}-17$ \\
\hline 20 & 1.0001163 & 1.0007919 & 1.0 & 0.9974290 & 0.9973274 & 0.9999609 & $1.102 \mathrm{E}-17$ \\
\hline 22 & 1.0001580 & 1.0007055 & 1.0 & 0.9974093 & 0.9975435 & 0.9999519 & $9.271 \mathrm{E}-18$ \\
\hline 24 & 1.0001782 & 0.9996965 & 1.0 & 0.9973497 & 0.9977324 & 0.9999524 & $7.977 \mathrm{E}-18$ \\
\hline 26 & 1.0001747 & 0.9996822 & 1.0 & 0.9974738 & 0.9978967 & 0.9999474 & $6.984 \mathrm{E}-18$ \\
\hline 28 & 1.0001876 & 1.0006199 & 1.0 & 0.9976103 & 0.9980685 & 0.9999508 & $6.196 \mathrm{E}-18$ \\
\hline 30 & 1.0001765 & 0.9998235 & 1.0 & 0.9974863 & 0.9981725 & 0.9999493 & $4.734 \mathrm{E}-18$ \\
\hline 32 & 1.0002152 & 1.0002538 & 1.0 & 0.9976880 & 0.9983247 & 0.9999464 & $4.266 \mathrm{E}-18$ \\
\hline 34 & 1.0002219 & 1.0010609 & 1.0 & 0.9976233 & 0.9984074 & 0.9999460 & $3.902 \mathrm{E}-18$ \\
\hline 36 & 1.0002177 & 1.0003426 & 1.0 & 0.9976737 & 0.9985103 & 0.9999538 & $3.571 \mathrm{E}-18$ \\
\hline 38 & 1.0002195 & 1.0000822 & 1.0 & 0.9977673 & 0.9985991 & 0.9999480 & $3.316 \mathrm{E}-18$ \\
\hline 40 & 1.0002196 & 0.9997804 & 1.0 & 0.9977829 & 0.9987117 & 0.9999522 & $2.828 \mathrm{E}-18$ \\
\hline 42 & 1.0002471 & 1.0005024 & 1.0 & 0.9977390 & 0.9988409 & 0.9999419 & $2.670 \mathrm{E}-18$ \\
\hline 44 & 1.0002448 & 1.0009426 & 1.0 & 0.9977765 & 0.9988952 & 0.9999538 & $2.529 \mathrm{E}-18$ \\
\hline 46 & 1.0002358 & 1.0009991 & 1.0 & 0.9977629 & 0.9989872 & 0.9999522 & $2.432 \mathrm{E}-18$ \\
\hline 48 & 1.0002475 & 1.0010392 & 1.0 & 0.9979882 & 0.9990457 & 0.9999486 & $2.334 \mathrm{E}-18$ \\
\hline 50 & 1.0002315 & 1.0002255 & 1.0000036 & 0.9978334 & 0.9991229 & 0.9999506 & $2.172 \mathrm{E}-18$ \\
\hline
\end{tabular}


b. Ritz chamber (cont'd).

\begin{tabular}{|c|c|c|c|c|c|c|c|}
\hline$E / \mathrm{keV}$ & $(1-g)^{-1}$ & $k_{\mathrm{eq}}$ & $k_{\mathrm{el}}$ & $k_{\mathrm{sc}}$ & $k_{\mathrm{fl}}$ & $k_{\mathrm{br}}$ & $P_{\text {rel }}$ \\
\hline 52 & 1.0002478 & 1.0011245 & 1.0000683 & 0.9977577 & 0.9992061 & 0.9999582 & $2.085 \mathrm{E}-18$ \\
\hline 54 & 1.0002291 & 0.9994704 & 1.0003007 & 0.9978193 & 0.9992720 & 0.9999563 & $2.038 \mathrm{E}-18$ \\
\hline 56 & 1.0002138 & 1.0001679 & 1.0011186 & 0.9978974 & 0.9993310 & 0.9999646 & $2.002 \mathrm{E}-18$ \\
\hline 58 & 1.0002026 & 1.0004479 & 1.0028882 & 0.9978886 & 0.9994012 & 0.9999637 & $1.984 \mathrm{E}-18$ \\
\hline 60 & 1.0002126 & 0.9995248 & 1.0058850 & 0.9980641 & 0.9994235 & 0.9999619 & $1.968 \mathrm{E}-18$ \\
\hline 62 & 1.0002041 & 1.0019827 & 1.0099604 & 0.9980151 & 0.9994735 & 0.9999633 & $1.913 \mathrm{E}-18$ \\
\hline 64 & 1.0002198 & 0.9994224 & 1.0152254 & 0.9980040 & 0.9995188 & 0.9999619 & $1.912 \mathrm{E}-18$ \\
\hline 66 & 1.0001914 & 1.0012683 & 1.0219123 & 0.9980829 & 0.9995702 & 0.9999653 & $1.924 \mathrm{E}-18$ \\
\hline 68 & 1.0002134 & 0.9996194 & 1.0288651 & 0.9981840 & 0.9995912 & 0.9999676 & $1.936 \mathrm{E}-18$ \\
\hline 70 & 1.0001747 & 1.0007563 & 1.0362296 & 0.9981151 & 0.9996009 & 0.9999580 & $1.952 \mathrm{E}-18$ \\
\hline 72 & 1.0002137 & 1.0005539 & 1.0436668 & 0.9981675 & 0.9996438 & 0.9999603 & $1.973 \mathrm{E}-18$ \\
\hline 74 & 1.0002108 & 1.0007109 & 1.0501383 & 0.9981279 & 0.9996798 & 0.9999586 & $1.995 \mathrm{E}-18$ \\
\hline 76 & 1.0002157 & 1.0000510 & 1.0566075 & 0.9980965 & 0.9996931 & 0.9999611 & $2.025 \mathrm{E}-18$ \\
\hline 78 & 1.0002004 & 0.9991657 & 1.0622996 & 0.9982181 & 0.9997184 & 0.9999643 & $2.050 \mathrm{E}-18$ \\
\hline 80 & 1.0001732 & 0.9998784 & 1.0665093 & 0.9981366 & 0.9997411 & 0.9999615 & $2.083 \mathrm{E}-18$ \\
\hline 82 & 1.0001922 & 0.9996665 & 1.0644509 & 0.9981675 & 0.9997723 & 0.9999629 & $2.069 \mathrm{E}-18$ \\
\hline 84 & 1.0001958 & 0.9991964 & 1.0655565 & 0.9982435 & 0.9997869 & 0.9999638 & $2.100 \mathrm{E}-18$ \\
\hline 86 & 1.0001971 & 1.0003183 & 1.0668154 & 0.9982933 & 0.9998000 & 0.9999599 & $2.139 \mathrm{E}-18$ \\
\hline 88 & 1.0001926 & 1.0000736 & 1.0682423 & 0.9983000 & 0.9998231 & 0.9999626 & $2.183 \mathrm{E}-18$ \\
\hline 90 & 1.0002114 & 0.9996883 & 1.0691059 & 0.9983129 & 0.9998289 & 0.9999608 & $2.231 \mathrm{E}-18$ \\
\hline 92 & 1.0002297 & 1.0001402 & 1.0691534 & 0.9983458 & 0.9998507 & 0.9999616 & $2.276 \mathrm{E}-18$ \\
\hline 94 & 1.0001832 & 1.0003445 & 1.0684333 & 0.9984353 & 0.9998598 & 0.9999562 & $2.324 \mathrm{E}-18$ \\
\hline 96 & 1.0002108 & 1.0005231 & 1.0672652 & 0.9983771 & 0.9998686 & 0.9999589 & $2.370 \mathrm{E}-18$ \\
\hline 98 & 1.0002106 & 1.0007573 & 1.0663093 & 0.9983969 & 0.9998776 & 0.9999623 & $2.425 \mathrm{E}-18$ \\
\hline 100 & 1.0002088 & 1.0008069 & 1.0612865 & 0.9984623 & 0.9998840 & 0.9999633 & $2.453 \mathrm{E}-18$ \\
\hline
\end{tabular}


c. Wyckoff-Attix chamber.

\begin{tabular}{|c|c|c|c|c|c|c|c|}
\hline$E / \mathrm{keV}$ & $(1-g)^{-1}$ & $k_{\mathrm{eq}}$ & $k_{\mathrm{el}}$ & $k_{\mathrm{sc}}$ & $k_{\mathrm{fl}}$ & $k_{\mathrm{br}}$ & $P_{\text {rel }}$ \\
\hline 10 & .0000330 & 9999670 & 1.0 & 0.9907690 & 9940463 & 0.99997 & 798E-17 \\
\hline 20 & 0001388 & .9998612 & 1.0 & .9931947 & 0.9965738 & 0.9999 & $1.547 \mathrm{E}-17$ \\
\hline 30 & 0001677 & .9999816 & 1.0 & .9936727 & 0.9976644 & 0.9999 & 6.699E-18 \\
\hline 40 & 0002682 & .9997319 & 1.0 & .9942618 & 0.9983533 & 0.9999264 & $4.032 \mathrm{E}-18$ \\
\hline 50 & 0002738 & .9997263 & 1.0 & 0.9948802 & 0.9988959 & 0.999 & $3.080 \mathrm{E}-18$ \\
\hline 60 & 0002159 & .9997841 & 1.0 & 0.9948538 & 0.9992142 & 0.999 & $2.807 \mathrm{E}-18$ \\
\hline 70 & 0001906 & 0.9987293 & 1.0 & 0.9953227 & 0.9995257 & 0.999 & $2.774 \mathrm{E}-18$ \\
\hline 80 & 0002135 & .0001245 & 1.0 & 0.9958322 & 0.9996827 & 0.999 & $2.959 \mathrm{E}-18$ \\
\hline 90 & 0002143 & .9999362 & 00016 & 0.9958384 & 0.9997936 & 0.999 & $3.174 \mathrm{E}-18$ \\
\hline 100 & 0002133 & 0.9994791 & 214 & 0.9965291 & 0.9998543 & 0.99 & $3.501 \mathrm{E}-18$ \\
\hline 110 & 0001829 & 0.9995988 & 2350 & 0.9966065 & 0.9998860 & 0.99 & $3.869 \mathrm{E}-18$ \\
\hline 120 & 0002313 & .9986789 & 648 & 0.9967747 & 9239 & 0.99 & $4.291 \mathrm{E}-18$ \\
\hline 130 & 0002146 & .9994416 & 823 & 0.9969651 & & 090 & $4.728 \mathrm{E}-18$ \\
\hline 140 & 0002621 & L.0006219 & 938 & 87 & 578 & 0.99 & $208 \mathrm{E}-18$ \\
\hline 150 & 0002698 & .9987145 & 2000 & 2348 & .9999717 & & -18 \\
\hline 160 & 0003211 & 0.9995951 & 800 & & & & $104 \mathrm{E}-18$ \\
\hline 170 & 0003654 & 0.9996044 & 178 & & & & $.574 \mathrm{E}-18$ \\
\hline 180 & & 1.0006345 & 1750 & & & & $.083 \mathrm{E}-18$ \\
\hline 190 & & & & & & & $.570 \mathrm{E}-18$ \\
\hline 200 & 49 & & {$[\Omega$} & & & & $.036 \mathrm{E}-18$ \\
\hline 210 & 57 & & ת & & & & $.550 \mathrm{E}-18$ \\
\hline 220 & Lu & & & & & & 9.049E-18 \\
\hline 230 & 0004783 & 0.9982574 &  & 129 & 9880 & 25 & 9.535E-18 \\
\hline 240 & 0004632 & 1.0014876 & 808 & 30811 & .9999882 & 60 & 1.005E-17 \\
\hline 250 & 391 & 19 & $\angle 0$. & 0923 & 903 & 70 & $1.054 \mathrm{E}-17$ \\
\hline 260 & 0005314 & 0.9999220 & - & 1757 & 0.9999895 & 47 & 1.102E-17 \\
\hline 270 & 0006003 & 0.998 & 322 & 98 & 9899 & 56 & 1.150E-17 \\
\hline 280 & 93 & 64 & 821 & & 920 & & 1.202E-17 \\
\hline 290 & 6469 & 0.997 & 7 & 2480 & 928 & 0.99 & 1.248E-17 \\
\hline 300 & 1.0006639 & 1.0016564 & 1.0899844 & 0.9983040 & 0.9999927 & 0.9999090 & 1.299E-17 \\
\hline
\end{tabular}


Table 4. Reconstructed data from Burns' Monte Carlo calculations for NIST FACs, using Eqs. (5). The quantities are defined in the text.

a. Lamperti chamber.

\begin{tabular}{|c|c|c|c|c|c|}
\hline$E / \mathrm{keV}$ & $P_{\text {dep }}$ & $P_{\text {col }}$ & $S_{\text {col }}$ & $F_{\text {col }}$ & $G_{\text {col }}$ \\
\hline 2 & $4.970 \mathrm{E}-19$ & $4.970 \mathrm{E}-19$ & $9.521 \mathrm{E}-22$ & 0 & 0 \\
\hline 4 & $8.651 \mathrm{E}-18$ & $8.651 \mathrm{E}-18$ & $2.639 \mathrm{E}-20$ & $5.059 \mathrm{E}-20$ & $3.561 \mathrm{E}-23$ \\
\hline 6 & $4.909 \mathrm{E}-18$ & $4.909 \mathrm{E}-18$ & $1.175 \mathrm{E}-20$ & $2.130 \mathrm{E}-20$ & 0 \\
\hline 8 & $3.014 \mathrm{E}-18$ & $3.014 \mathrm{E}-18$ & $5.759 \mathrm{E}-21$ & $1.077 \mathrm{E}-20$ & $1.386 \mathrm{E}-23$ \\
\hline 10 & $1.701 \mathrm{E}-18$ & $1.701 \mathrm{E}-18$ & $2.917 \mathrm{E}-21$ & $5.086 \mathrm{E}-21$ & $2.217 \mathrm{E}-23$ \\
\hline 12 & $1.247 \mathrm{E}-18$ & $1.247 \mathrm{E}-18$ & $1.632 \mathrm{E}-21$ & $3.315 \mathrm{E}-21$ & $2.347 \mathrm{E}-23$ \\
\hline 14 & $9.803 \mathrm{E}-19$ & $9.803 \mathrm{E}-19$ & $1.444 \mathrm{E}-21$ & $2.378 \mathrm{E}-21$ & $1.172 \mathrm{E}-23$ \\
\hline 16 & $6.510 \mathrm{E}-19$ & $6.510 \mathrm{E}-19$ & $8.603 \mathrm{E}-22$ & $1.365 \mathrm{E}-21$ & $2.206 \mathrm{E}-23$ \\
\hline 18 & $5.307 \mathrm{E}-19$ & $5.307 \mathrm{E}-19$ & $6.222 \mathrm{E}-22$ & $1.031 \mathrm{E}-21$ & $1.976 \mathrm{E}-23$ \\
\hline 20 & $3.958 \mathrm{E}-19$ & $3.958 \mathrm{E}-19$ & $4.274 \mathrm{E}-22$ & $6.961 \mathrm{E}-22$ & $1.373 \mathrm{E}-23$ \\
\hline 22 & $3.342 \mathrm{E}-19$ & $3.342 \mathrm{E}-19$ & $3.554 \mathrm{E}-22$ & $5.206 \mathrm{E}-22$ & $9.124 \mathrm{E}-24$ \\
\hline 24 & $2.889 \mathrm{E}-19$ & $2.889 \mathrm{E}-19$ & $2.792 \mathrm{E}-22$ & $4.167 \mathrm{E}-22$ & $9.574 \mathrm{E}-24$ \\
\hline 26 & $2.517 \mathrm{E}-19$ & $2.517 \mathrm{E}-19$ & $2.545 \mathrm{E}-22$ & $3.340 \mathrm{E}-22$ & $8.836 \mathrm{E}-24$ \\
\hline 28 & $2.232 \mathrm{E}-19$ & $2.232 \mathrm{E}-19$ & $2.499 \mathrm{E}-22$ & $2.875 \mathrm{E}-22$ & $6.020 \mathrm{E}-24$ \\
\hline 30 & $1.707 \mathrm{E}-19$ & $1.707 \mathrm{E}-19$ & $2.176 \mathrm{E}-22$ & $1.896 \mathrm{E}-22$ & $8.321 \mathrm{E}-24$ \\
\hline 32 & $1.527 \mathrm{E}-19$ & $1.527 \mathrm{E}-19$ & $1.432 \mathrm{E}-22$ & $1.777 \mathrm{E}-22$ & $5.891 \mathrm{E}-24$ \\
\hline 34 & $1.407 \mathrm{E}-19$ & $1.407 \mathrm{E}-19$ & $1.333 \mathrm{E}-22$ & $1.385 \mathrm{E}-22$ & $3.484 \mathrm{E}-24$ \\
\hline 36 & $1.279 \mathrm{E}-19$ & $1.277 \mathrm{E}-19$ & $1.315 \mathrm{E}-22$ & $1.233 \mathrm{E}-22$ & $7.159 \mathrm{E}-24$ \\
\hline 38 & $1.194 \mathrm{E}-19$ & $1.187 \mathrm{E}-19$ & $1.185 \mathrm{E}-22$ & $1.056 \mathrm{E}-22$ & $3.676 \mathrm{E}-24$ \\
\hline 40 & $1.016 \mathrm{E}-19$ & $1.002 \mathrm{E}-19$ & $1.284 \mathrm{E}-22$ & $8.090 \mathrm{E}-23$ & $3.592 \mathrm{E}-24$ \\
\hline 42 & $9.628 \mathrm{E}-20$ & $9.377 \mathrm{E}-20$ & $8.391 \mathrm{E}-23$ & $7.028 \mathrm{E}-23$ & $3.204 \mathrm{E}-24$ \\
\hline 44 & $9.117 \mathrm{E}-20$ & $8.728 \mathrm{E}-20$ & $8.430 \mathrm{E}-23$ & $6.260 \mathrm{E}-23$ & $3.160 \mathrm{E}-24$ \\
\hline 46 & $8.722 \mathrm{E}-20$ & $8.150 \mathrm{E}-20$ & $8.218 \mathrm{E}-23$ & $5.506 \mathrm{E}-23$ & $3.362 \mathrm{E}-24$ \\
\hline 48 & $8.436 \mathrm{E}-20$ & $7.681 \mathrm{E}-20$ & $7.568 \mathrm{E}-23$ & $5.429 \mathrm{E}-23$ & $3.341 \mathrm{E}-24$ \\
\hline 50 & $7.822 \mathrm{E}-20$ & $6.952 \mathrm{E}-20$ & $8.337 \mathrm{E}-23$ & $4.604 \mathrm{E}-23$ & $2.770 \mathrm{E}-24$ \\
\hline
\end{tabular}


b. Ritz chamber.

\begin{tabular}{|c|c|c|c|c|c|}
\hline$E / k e V$ & $P_{\text {dep }}$ & $P_{\text {col }}$ & $S_{\text {col }}$ & $F_{\text {col }}$ & $G_{\text {col }}$ \\
\hline 2 & $1.156 \mathrm{E}-21$ & $1.156 \mathrm{E}-21$ & 0 & 0 & 0 \\
\hline 4 & $9.746 \mathrm{E}-17$ & $9.746 \mathrm{E}-17$ & $5.996 \mathrm{E}-19$ & $8.686 \mathrm{E}-19$ & $3.981 \mathrm{E}-23$ \\
\hline 6 & $1.052 \mathrm{E}-16$ & $1.052 \mathrm{E}-16$ & $5.537 \mathrm{E}-19$ & $6.991 \mathrm{E}-19$ & 0 \\
\hline 8 & $7.487 \mathrm{E}-17$ & $7.487 \mathrm{E}-17$ & $3.481 \mathrm{E}-19$ & $4.119 \mathrm{E}-19$ & $4.656 \mathrm{E}-23$ \\
\hline 10 & $4.523 \mathrm{E}-17$ & $4.523 \mathrm{E}-17$ & $1.694 \mathrm{E}-19$ & $2.129 \mathrm{E}-19$ & $7.375 \mathrm{E}-22$ \\
\hline 12 & $3.365 \mathrm{E}-17$ & $3.365 \mathrm{E}-17$ & $1.147 \mathrm{E}-19$ & $1.376 \mathrm{E}-19$ & $6.186 \mathrm{E}-22$ \\
\hline 14 & $2.653 \mathrm{E}-17$ & $2.653 \mathrm{E}-17$ & $8.945 \mathrm{E}-20$ & $9.292 \mathrm{E}-20$ & $6.771 \mathrm{E}-22$ \\
\hline 16 & $1.790 \mathrm{E}-17$ & $1.790 \mathrm{E}-17$ & $4.818 \mathrm{E}-20$ & $5.665 \mathrm{E}-20$ & $6.187 \mathrm{E}-22$ \\
\hline 18 & $1.464 \mathrm{E}-17$ & $1.464 \mathrm{E}-17$ & $4.038 \mathrm{E}-20$ & $4.332 \mathrm{E}-20$ & $6.447 \mathrm{E}-22$ \\
\hline 20 & $1.101 \mathrm{E}-17$ & $1.101 \mathrm{E}-17$ & $2.838 \mathrm{E}-20$ & $2.958 \mathrm{E}-20$ & $4.331 \mathrm{E}-22$ \\
\hline 22 & $9.263 \mathrm{E}-18$ & $9.263 \mathrm{E}-18$ & $2.406 \mathrm{E}-20$ & $2.287 \mathrm{E}-20$ & $4.478 \mathrm{E}-22$ \\
\hline 24 & $7.978 \mathrm{E}-18$ & $7.978 \mathrm{E}-18$ & $2.120 \mathrm{E}-20$ & $1.818 \mathrm{E}-20$ & $3.815 \mathrm{E}-22$ \\
\hline 26 & $6.985 \mathrm{E}-18$ & $6.985 \mathrm{E}-18$ & $1.769 \mathrm{E}-20$ & $1.476 \mathrm{E}-20$ & $3.692 \mathrm{E}-22$ \\
\hline 28 & $6.191 \mathrm{E}-18$ & $6.191 \mathrm{E}-18$ & $1.483 \mathrm{E}-20$ & $1.201 \mathrm{E}-20$ & $3.060 \mathrm{E}-22$ \\
\hline 30 & $4.734 \mathrm{E}-18$ & $4.734 \mathrm{E}-18$ & $1.193 \mathrm{E}-20$ & $8.689 \mathrm{E}-21$ & $2.411 \mathrm{E}-22$ \\
\hline 32 & $4.264 \mathrm{E}-18$ & $4.264 \mathrm{E}-18$ & $9.881 \mathrm{E}-21$ & $7.172 \mathrm{E}-21$ & $2.293 \mathrm{E}-22$ \\
\hline 34 & $3.897 \mathrm{E}-18$ & $3.897 \mathrm{E}-18$ & $9.284 \mathrm{E}-21$ & $6.231 \mathrm{E}-21$ & $2.113 \mathrm{E}-22$ \\
\hline 36 & $3.569 \mathrm{E}-18$ & $3.569 \mathrm{E}-18$ & $8.322 \mathrm{E}-21$ & $5.337 \mathrm{E}-21$ & $1.656 \mathrm{E}-22$ \\
\hline 38 & $3.315 \mathrm{E}-18$ & $3.315 \mathrm{E}-18$ & $7.418 \mathrm{E}-21$ & $4.661 \mathrm{E}-21$ & $1.731 \mathrm{E}-22$ \\
\hline 40 & $2.828 \mathrm{E}-18$ & $2.828 \mathrm{E}-18$ & $6.284 \mathrm{E}-21$ & $3.656 \mathrm{E}-21$ & $1.356 \mathrm{E}-22$ \\
\hline 42 & $2.668 \mathrm{E}-18$ & $2.668 \mathrm{E}-18$ & $6.046 \mathrm{E}-21$ & $3.103 \mathrm{E}-21$ & $1.555 \mathrm{E}-22$ \\
\hline 44 & $2.526 \mathrm{E}-18$ & $2.526 \mathrm{E}-18$ & $5.629 \mathrm{E}-21$ & $2.800 \mathrm{E}-21$ & $1.170 \mathrm{E}-22$ \\
\hline 46 & $2.429 \mathrm{E}-18$ & $2.429 \mathrm{E}-18$ & $5.446 \mathrm{E}-21$ & $2.468 \mathrm{E}-21$ & $1.164 \mathrm{E}-22$ \\
\hline 48 & $2.331 \mathrm{E}-18$ & $2.331 \mathrm{E}-18$ & $4.699 \mathrm{E}-21$ & $2.231 \mathrm{E}-21$ & $1.201 \mathrm{E}-22$ \\
\hline 50 & $2.171 \mathrm{E}-18$ & $2.171 \mathrm{E}-18$ & $4.714 \mathrm{E}-21$ & $1.910 \mathrm{E}-21$ & $1.076 \mathrm{E}-22$ \\
\hline
\end{tabular}


b. Ritz chamber (cont’d).

\begin{tabular}{|c|c|c|c|c|c|}
\hline$E / k e V$ & $P_{\text {dep }}$ & $P_{\text {col }}$ & $S_{\text {col }}$ & $F_{\text {col }}$ & $G_{\text {col }}$ \\
\hline 52 & $2.082 \mathrm{E}-18$ & $2.082 \mathrm{E}-18$ & $4.678 \mathrm{E}-21$ & $1.487 \mathrm{E}-21$ & $8.718 \mathrm{E}-23$ \\
\hline 54 & $2.039 \mathrm{E}-18$ & $2.037 \mathrm{E}-18$ & $4.452 \mathrm{E}-21$ & $1.340 \mathrm{E}-21$ & $8.927 \mathrm{E}-23$ \\
\hline 56 & $2.001 \mathrm{E}-18$ & $1.998 \mathrm{E}-18$ & $4.210 \mathrm{E}-21$ & $1.187 \mathrm{E}-21$ & $7.088 \mathrm{E}-23$ \\
\hline 58 & $1.983 \mathrm{E}-18$ & $1.976 \mathrm{E}-18$ & $4.182 \mathrm{E}-21$ & $1.131 \mathrm{E}-21$ & $7.186 \mathrm{E}-23$ \\
\hline 60 & $1.969 \mathrm{E}-18$ & $1.957 \mathrm{E}-18$ & $3.796 \mathrm{E}-21$ & $9.978 \mathrm{E}-22$ & $7.484 \mathrm{E}-23$ \\
\hline 62 & $1.909 \mathrm{E}-18$ & $1.890 \mathrm{E}-18$ & $3.760 \mathrm{E}-21$ & $9.090 \mathrm{E}-22$ & $6.959 \mathrm{E}-23$ \\
\hline 64 & $1.913 \mathrm{E}-18$ & $1.884 \mathrm{E}-18$ & $3.769 \mathrm{E}-21$ & $8.104 \mathrm{E}-22$ & $7.193 \mathrm{E}-23$ \\
\hline 66 & $1.921 \mathrm{E}-18$ & $1.881 \mathrm{E}-18$ & $3.613 \mathrm{E}-21$ & $7.712 \mathrm{E}-22$ & $6.534 \mathrm{E}-23$ \\
\hline 68 & $1.936 \mathrm{E}-18$ & $1.882 \mathrm{E}-18$ & $3.424 \mathrm{E}-21$ & $7.527 \mathrm{E}-22$ & $6.116 \mathrm{E}-23$ \\
\hline 70 & $1.950 \mathrm{E}-18$ & $1.882 \mathrm{E}-18$ & $3.553 \mathrm{E}-21$ & $6.741 \mathrm{E}-22$ & $7.914 \mathrm{E}-23$ \\
\hline 72 & $1.971 \mathrm{E}-18$ & $1.888 \mathrm{E}-18$ & $3.467 \mathrm{E}-21$ & $6.086 \mathrm{E}-22$ & $7.516 \mathrm{E}-23$ \\
\hline 74 & $1.993 \mathrm{E}-18$ & $1.897 \mathrm{E}-18$ & $3.557 \mathrm{E}-21$ & $5.893 \mathrm{E}-22$ & $7.876 \mathrm{E}-23$ \\
\hline 76 & $2.024 \mathrm{E}-18$ & $1.916 \mathrm{E}-18$ & $3.654 \mathrm{E}-21$ & $5.454 \mathrm{E}-22$ & $7.462 \mathrm{E}-23$ \\
\hline 78 & $2.051 \mathrm{E}-18$ & $1.933 \mathrm{E}-18$ & $3.451 \mathrm{E}-21$ & $5.077 \mathrm{E}-22$ & $6.919 \mathrm{E}-23$ \\
\hline 80 & $2.083 \mathrm{E}-18$ & $1.957 \mathrm{E}-18$ & $3.653 \mathrm{E}-21$ & $4.427 \mathrm{E}-22$ & $7.557 \mathrm{E}-23$ \\
\hline 82 & $2.069 \mathrm{E}-18$ & $1.940 \mathrm{E}-18$ & $3.562 \mathrm{E}-21$ & $4.203 \mathrm{E}-22$ & $7.206 \mathrm{E}-23$ \\
\hline 84 & $2.101 \mathrm{E}-18$ & $1.968 \mathrm{E}-18$ & $3.463 \mathrm{E}-21$ & $4.011 \mathrm{E}-22$ & $7.148 \mathrm{E}-23$ \\
\hline 86 & $2.138 \mathrm{E}-18$ & $2.002 \mathrm{E}-18$ & $3.422 \mathrm{E}-21$ & $3.620 \mathrm{E}-22$ & $8.036 \mathrm{E}-23$ \\
\hline 88 & $2.182 \mathrm{E}-18$ & $2.043 \mathrm{E}-18$ & $3.479 \mathrm{E}-21$ & $3.581 \mathrm{E}-22$ & $7.653 \mathrm{E}-23$ \\
\hline 90 & $2.231 \mathrm{E}-18$ & $2.089 \mathrm{E}-18$ & $3.530 \mathrm{E}-21$ & $3.185 \mathrm{E}-22$ & $8.205 \mathrm{E}-23$ \\
\hline 92 & $2.275 \mathrm{E}-18$ & $2.130 \mathrm{E}-18$ & $3.529 \mathrm{E}-21$ & $3.055 \mathrm{E}-22$ & $8.190 \mathrm{E}-23$ \\
\hline 94 & $2.323 \mathrm{E}-18$ & $2.175 \mathrm{E}-18$ & $3.409 \mathrm{E}-21$ & $2.921 \mathrm{E}-22$ & $9.552 \mathrm{E}-23$ \\
\hline 96 & $2.368 \mathrm{E}-18$ & $2.220 \mathrm{E}-18$ & $3.608 \mathrm{E}-21$ & $2.788 \mathrm{E}-22$ & $9.143 \mathrm{E}-23$ \\
\hline 98 & $2.423 \mathrm{E}-18$ & $2.274 \mathrm{E}-18$ & $3.652 \mathrm{E}-21$ & $2.681 \mathrm{E}-22$ & $8.583 \mathrm{E}-23$ \\
\hline 100 & $2.451 \mathrm{E}-18$ & $2.307 \mathrm{E}-18$ & $3.553 \mathrm{E}-21$ & $1.487 \mathrm{E}-21$ & $8.474 \mathrm{E}-23$ \\
\hline
\end{tabular}


c. Wyckoff-Attix chamber.

\begin{tabular}{|c|c|c|c|c|c|}
\hline$E / \mathrm{keV}$ & $P_{\text {dep }}$ & $P_{\mathrm{col}}$ & $S_{\mathrm{col}}$ & $F_{\text {col }}$ & $G_{\mathrm{col}}$ \\
\hline 10 & 5.798E-17 & $5.798 \mathrm{E}-17$ & 5.402E-19 & 3.505E-19 & $1.248 \mathrm{E}-21$ \\
\hline 20 & 1.547E-17 & 1.547E-17 & 1.060E-19 & $5.355 \mathrm{E}-20$ & $9.189 \mathrm{E}-22$ \\
\hline 30 & $6.698 \mathrm{E}-18$ & $6.698 \mathrm{E}-18$ & $4.265 \mathrm{E}-20$ & 1.578E-20 & 4.982E-22 \\
\hline 40 & $4.032 \mathrm{E}-18$ & $4.032 \mathrm{E}-18$ & $2.327 \mathrm{E}-20$ & $6.689 \mathrm{E}-21$ & $2.988 \mathrm{E}-22$ \\
\hline 50 & $3.080 \mathrm{E}-18$ & 3.080E-18 & 1.585E-20 & $3.422 \mathrm{E}-21$ & $1.875 \mathrm{E}-22$ \\
\hline 60 & $2.807 \mathrm{E}-18$ & 2.807E-18 & $1.452 \mathrm{E}-20$ & $2.219 \mathrm{E}-21$ & $1.436 \mathrm{E}-22$ \\
\hline 70 & $2.777 \mathrm{E}-18$ & $2.777 \mathrm{E}-18$ & $1.305 \mathrm{E}-20$ & $1.324 \mathrm{E}-21$ & $1.282 \mathrm{E}-22$ \\
\hline 80 & $2.958 \mathrm{E}-18$ & $2.958 \mathrm{E}-18$ & $1.238 \mathrm{E}-20$ & $9.428 \mathrm{E}-22$ & $1.338 \mathrm{E}-22$ \\
\hline 90 & $3.174 \mathrm{E}-18$ & $3.173 \mathrm{E}-18$ & 1.326E-20 & $6.578 \mathrm{E}-22$ & 1.807E-22 \\
\hline 100 & 3.502E-18 & 3.497E-18 & $1.218 \mathrm{E}-20$ & $5.115 \mathrm{E}-22$ & $2.229 \mathrm{E}-22$ \\
\hline 110 & $3.870 \mathrm{E}-18$ & 3.856E-18 & 1.313E-20 & $4.410 \mathrm{E}-22$ & $2.266 \mathrm{E}-22$ \\
\hline 120 & $4.296 \mathrm{E}-18$ & $4.268 \mathrm{E}-18$ & 1.381E-20 & $3.257 \mathrm{E}-22$ & 2.494E-22 \\
\hline 130 & $4.730 \mathrm{E}-18$ & 4.691E-18 & $1.428 \mathrm{E}-20$ & $2.850 \mathrm{E}-22$ & $2.896 \mathrm{E}-22$ \\
\hline 140 & $5.203 \mathrm{E}-18$ & 5.155E-18 & $1.557 \mathrm{E}-20$ & $2.180 \mathrm{E}-22$ & $3.022 \mathrm{E}-22$ \\
\hline 150 & $5.630 \mathrm{E}-18$ & 5.579E-18 & 1.547E-20 & $1.586 \mathrm{E}-22$ & 3.859E-22 \\
\hline 160 & $6.105 \mathrm{E}-18$ & $6.054 \mathrm{E}-18$ & 1.609E-20 & $1.714 \mathrm{E}-22$ & $4.691 \mathrm{E}-22$ \\
\hline 170 & $6.574 \mathrm{E}-18$ & $6.523 \mathrm{E}-18$ & $1.635 \mathrm{E}-20$ & 1.894E-22 & $4.880 \mathrm{E}-22$ \\
\hline 180 & 7.076E-18 & 7.022E-18 & 1.751E-20 & 1.467E-22 & $5.222 \mathrm{E}-22$ \\
\hline 190 & $7.566 \mathrm{E}-18$ & $7.513 \mathrm{E}-18$ & 1.804E-20 & $1.302 \mathrm{E}-22$ & $5.508 \mathrm{E}-22$ \\
\hline 200 & $8.033 \mathrm{E}-18$ & 7.986E-18 & 1.809E-20 & 1.118E-22 & $5.834 \mathrm{E}-22$ \\
\hline 210 & 8.546E-18 & $8.501 \mathrm{E}-18$ & 1.865E-20 & 1.227E-22 & 6.444E-22 \\
\hline 220 & $9.040 \mathrm{E}-18$ & 8.993E-18 & $1.918 \mathrm{E}-20$ & 1.199E-22 & $6.877 \mathrm{E}-22$ \\
\hline 230 & $9.547 \mathrm{E}-18$ & $9.491 \mathrm{E}-18$ & 1.985E-20 & 1.140E-22 & $7.375 \mathrm{E}-22$ \\
\hline 240 & 1.003E-17 & $9.950 \mathrm{E}-18$ & 1.913E-20 & 1.177E-22 & 8.378E-22 \\
\hline 250 & $1.051 \mathrm{E}-17$ & $1.038 \mathrm{E}-17$ & 1.984E-20 & 1.013E-22 & $8.633 \mathrm{E}-22$ \\
\hline 260 & 1.102E-17 & $1.079 \mathrm{E}-17$ & $1.972 \mathrm{E}-20$ & 1.139E-22 & $9.217 \mathrm{E}-22$ \\
\hline 270 & 1.151E-17 & 1.115E-17 & $2.022 \mathrm{E}-20$ & 1.133E-22 & $9.429 \mathrm{E}-22$ \\
\hline 280 & 1.199E-17 & 1.144E-17 & $2.076 \mathrm{E}-20$ & $9.174 \mathrm{E}-23$ & $8.876 \mathrm{E}-22$ \\
\hline 290 & $1.251 \mathrm{E}-17$ & $1.172 \mathrm{E}-17$ & $2.057 \mathrm{E}-20$ & 8.445E-23 & $9.501 \mathrm{E}-22$ \\
\hline 300 & $1.296 \mathrm{E}-17$ & 1.189E-17 & 2.020E-20 & 8.681E-23 & 1.084E-21 \\
\hline
\end{tabular}


Table 5. The adopted, smoothed data from Burns' Monte Carlo calculations for NIST FACs.

a. Lamperti chamber.

\begin{tabular}{|c|c|c|c|c|c|}
\hline$E / k e V$ & $P_{\text {dep }}$ & $P_{\text {col }}$ & $S_{\text {col }}$ & $F_{\text {col }}$ & $G_{\text {col }}$ \\
\hline 2 & $1.539 \mathrm{E}-17$ & $1.539 \mathrm{E}-17$ & $7.104 \mathrm{E}-20$ & $1.272 \mathrm{E}-19$ & $3.256 \mathrm{E}-23$ \\
\hline 4 & $8.684 \mathrm{E}-18$ & $8.684 \mathrm{E}-18$ & $2.666 \mathrm{E}-20$ & $4.921 \mathrm{E}-20$ & $2.872 \mathrm{E}-23$ \\
\hline 6 & $4.891 \mathrm{E}-18$ & $4.891 \mathrm{E}-18$ & $1.152 \mathrm{E}-20$ & $2.159 \mathrm{E}-20$ & $2.567 \mathrm{E}-23$ \\
\hline 8 & $2.901 \mathrm{E}-18$ & $2.901 \mathrm{E}-18$ & $5.638 \mathrm{E}-21$ & $1.058 \mathrm{E}-20$ & $2.313 \mathrm{E}-23$ \\
\hline 10 & $1.842 \mathrm{E}-18$ & $1.842 \mathrm{E}-18$ & $3.073 \mathrm{E}-21$ & $5.706 \mathrm{E}-21$ & $2.096 \mathrm{E}-23$ \\
\hline 12 & $1.249 \mathrm{E}-18$ & $1.249 \mathrm{E}-18$ & $1.840 \mathrm{E}-21$ & $3.343 \mathrm{E}-21$ & $1.902 \mathrm{E}-23$ \\
\hline 14 & $8.955 \mathrm{E}-19$ & $8.955 \mathrm{E}-19$ & $1.193 \mathrm{E}-21$ & $2.101 \mathrm{E}-21$ & $1.726 \mathrm{E}-23$ \\
\hline 16 & $6.718 \mathrm{E}-19$ & $6.718 \mathrm{E}-19$ & $8.274 \mathrm{E}-22$ & $1.402 \mathrm{E}-21$ & $1.562 \mathrm{E}-23$ \\
\hline 18 & $5.222 \mathrm{E}-19$ & $5.222 \mathrm{E}-19$ & $6.071 \mathrm{E}-22$ & $9.822 \mathrm{E}-22$ & $1.408 \mathrm{E}-23$ \\
\hline 20 & $4.176 \mathrm{E}-19$ & $4.176 \mathrm{E}-19$ & $4.665 \mathrm{E}-22$ & $7.166 \mathrm{E}-22$ & $1.262 \mathrm{E}-23$ \\
\hline 22 & $3.419 \mathrm{E}-19$ & $3.419 \mathrm{E}-19$ & $3.720 \mathrm{E}-22$ & $5.400 \mathrm{E}-22$ & $1.125 \mathrm{E}-23$ \\
\hline 24 & $2.854 \mathrm{E}-19$ & $2.854 \mathrm{E}-19$ & $3.055 \mathrm{E}-22$ & $4.175 \mathrm{E}-22$ & $9.978 \mathrm{E}-24$ \\
\hline 26 & $2.422 \mathrm{E}-19$ & $2.422 \mathrm{E}-19$ & $2.566 \mathrm{E}-22$ & $3.292 \mathrm{E}-22$ & $8.801 \mathrm{E}-24$ \\
\hline 28 & $2.084 \mathrm{E}-19$ & $2.084 \mathrm{E}-19$ & $2.193 \mathrm{E}-22$ & $2.636 \mathrm{E}-22$ & $7.730 \mathrm{E}-24$ \\
\hline 30 & $1.814 \mathrm{E}-19$ & $1.814 \mathrm{E}-19$ & $1.898 \mathrm{E}-22$ & $2.135 \mathrm{E}-22$ & $6.772 \mathrm{E}-24$ \\
\hline 32 & $1.593 \mathrm{E}-19$ & $1.593 \mathrm{E}-19$ & $1.659 \mathrm{E}-22$ & $1.745 \mathrm{E}-22$ & $5.930 \mathrm{E}-24$ \\
\hline 34 & $1.411 \mathrm{E}-19$ & $1.411 \mathrm{E}-19$ & $1.461 \mathrm{E}-22$ & $1.437 \mathrm{E}-22$ & $5.202 \mathrm{E}-24$ \\
\hline 36 & $1.261 \mathrm{E}-19$ & $1.259 \mathrm{E}-19$ & $1.295 \mathrm{E}-22$ & $1.192 \mathrm{E}-22$ & $4.586 \mathrm{E}-24$ \\
\hline 38 & $1.139 \mathrm{E}-19$ & $1.132 \mathrm{E}-19$ & $1.156 \mathrm{E}-22$ & $9.964 \mathrm{E}-23$ & $4.078 \mathrm{E}-24$ \\
\hline 40 & $1.042 \mathrm{E}-19$ & $1.028 \mathrm{E}-19$ & $1.041 \mathrm{E}-22$ & $8.413 \mathrm{E}-23$ & $3.673 \mathrm{E}-24$ \\
\hline 42 & $9.679 \mathrm{E}-20$ & $9.431 \mathrm{E}-20$ & $9.470 \mathrm{E}-23$ & $7.193 \mathrm{E}-23$ & $3.365 \mathrm{E}-24$ \\
\hline 44 & $9.142 \mathrm{E}-20$ & $8.747 \mathrm{E}-20$ & $8.745 \mathrm{E}-23$ & $6.253 \mathrm{E}-23$ & $3.152 \mathrm{E}-24$ \\
\hline 46 & $8.742 \mathrm{E}-20$ & $8.171 \mathrm{E}-20$ & $8.234 \mathrm{E}-23$ & $5.555 \mathrm{E}-23$ & $3.036 \mathrm{E}-24$ \\
\hline 48 & $8.368 \mathrm{E}-20$ & $7.619 \mathrm{E}-20$ & $7.954 \mathrm{E}-23$ & $5.073 \mathrm{E}-23$ & $3.026 \mathrm{E}-24$ \\
\hline 50 & $7.844 \mathrm{E}-20$ & $6.972 \mathrm{E}-20$ & $7.938 \mathrm{E}-23$ & $4.798 \mathrm{E}-23$ & $3.140 \mathrm{E}-24$ \\
\hline
\end{tabular}


b. Ritz chamber.

\begin{tabular}{|c|c|c|c|c|c|}
\hline$E / k e V$ & $P_{\text {dep }}$ & $P_{\text {col }}$ & $S_{\text {col }}$ & $F_{\text {col }}$ & $G_{\text {col }}$ \\
\hline 2 & $2.608 \mathrm{E}-16$ & $2.608 \mathrm{E}-16$ & $2.206 \mathrm{E}-18$ & $2.994 \mathrm{E}-18$ & $7.171 \mathrm{E}-22$ \\
\hline 4 & $1.663 \mathrm{E}-16$ & $1.663 \mathrm{E}-16$ & $1.104 \mathrm{E}-18$ & $1.411 \mathrm{E}-18$ & $7.503 \mathrm{E}-22$ \\
\hline 6 & $1.071 \mathrm{E}-16$ & $1.071 \mathrm{E}-16$ & $5.738 \mathrm{E}-19$ & $7.147 \mathrm{E}-19$ & $7.623 \mathrm{E}-22$ \\
\hline 8 & $7.064 \mathrm{E}-17$ & $7.064 \mathrm{E}-17$ & $3.156 \mathrm{E}-19$ & $3.876 \mathrm{E}-19$ & $7.547 \mathrm{E}-22$ \\
\hline 10 & $4.807 \mathrm{E}-17$ & $4.807 \mathrm{E}-17$ & $1.851 \mathrm{E}-19$ & $2.240 \mathrm{E}-19$ & $7.306 \mathrm{E}-22$ \\
\hline 12 & $3.386 \mathrm{E}-17$ & $3.386 \mathrm{E}-17$ & $1.159 \mathrm{E}-19$ & $1.372 \mathrm{E}-19$ & $6.935 \mathrm{E}-22$ \\
\hline 14 & $2.470 \mathrm{E}-17$ & $2.470 \mathrm{E}-17$ & $7.715 \mathrm{E}-20$ & $8.860 \mathrm{E}-20$ & $6.474 \mathrm{E}-22$ \\
\hline 16 & $1.862 \mathrm{E}-17$ & $1.862 \mathrm{E}-17$ & $5.428 \mathrm{E}-20$ & $5.993 \mathrm{E}-20$ & $5.958 \mathrm{E}-22$ \\
\hline 18 & $1.447 \mathrm{E}-17$ & $1.447 \mathrm{E}-17$ & $4.005 \mathrm{E}-20$ & $4.222 \mathrm{E}-20$ & $5.419 \mathrm{E}-22$ \\
\hline 20 & $1.155 \mathrm{E}-17$ & $1.155 \mathrm{E}-17$ & $3.077 \mathrm{E}-20$ & $3.081 \mathrm{E}-20$ & $4.882 \mathrm{E}-22$ \\
\hline 22 & $9.439 \mathrm{E}-18$ & $9.439 \mathrm{E}-18$ & $2.443 \mathrm{E}-20$ & $2.316 \mathrm{E}-20$ & $4.365 \mathrm{E}-22$ \\
\hline 24 & $7.868 \mathrm{E}-18$ & $7.868 \mathrm{E}-18$ & $1.992 \mathrm{E}-20$ & $1.785 \mathrm{E}-20$ & $3.882 \mathrm{E}-22$ \\
\hline 26 & $6.673 \mathrm{E}-18$ & $6.673 \mathrm{E}-18$ & $1.659 \mathrm{E}-20$ & $1.406 \mathrm{E}-20$ & $3.439 \mathrm{E}-22$ \\
\hline 28 & $5.742 \mathrm{E}-18$ & $5.742 \mathrm{E}-18$ & $1.406 \mathrm{E}-20$ & $1.126 \mathrm{E}-20$ & $3.040 \mathrm{E}-22$ \\
\hline 30 & $5.005 \mathrm{E}-18$ & $5.005 \mathrm{E}-18$ & $1.209 \mathrm{E}-20$ & $9.157 \mathrm{E}-21$ & $2.685 \mathrm{E}-22$ \\
\hline 32 & $4.412 \mathrm{E}-18$ & $4.412 \mathrm{E}-18$ & $1.052 \mathrm{E}-20$ & $7.537 \mathrm{E}-21$ & $2.374 \mathrm{E}-22$ \\
\hline 34 & $3.930 \mathrm{E}-18$ & $3.930 \mathrm{E}-18$ & $9.253 \mathrm{E}-21$ & $6.268 \mathrm{E}-21$ & $2.103 \mathrm{E}-22$ \\
\hline 36 & $3.535 \mathrm{E}-18$ & $3.535 \mathrm{E}-18$ & $8.222 \mathrm{E}-21$ & $5.260 \mathrm{E}-21$ & $1.869 \mathrm{E}-22$ \\
\hline 38 & $3.209 \mathrm{E}-18$ & $3.209 \mathrm{E}-18$ & $7.378 \mathrm{E}-21$ & $4.449 \mathrm{E}-21$ & $1.668 \mathrm{E}-22$ \\
\hline 40 & $2.941 \mathrm{E}-18$ & $2.941 \mathrm{E}-18$ & $6.684 \mathrm{E}-21$ & $3.790 \mathrm{E}-21$ & $1.496 \mathrm{E}-22$ \\
\hline 42 & $2.719 \mathrm{E}-18$ & $2.719 \mathrm{E}-18$ & $6.113 \mathrm{E}-21$ & $3.251 \mathrm{E}-21$ & $1.350 \mathrm{E}-22$ \\
\hline 44 & $2.537 \mathrm{E}-18$ & $2.537 \mathrm{E}-18$ & $5.644 \mathrm{E}-21$ & $2.808 \mathrm{E}-21$ & $1.226 \mathrm{E}-22$ \\
\hline 46 & $2.388 \mathrm{E}-18$ & $2.388 \mathrm{E}-18$ & $5.258 \mathrm{E}-21$ & $2.441 \mathrm{E}-21$ & $1.121 \mathrm{E}-22$ \\
\hline 48 & $2.267 \mathrm{E}-18$ & $2.267 \mathrm{E}-18$ & $4.942 \mathrm{E}-21$ & $2.136 \mathrm{E}-21$ & $1.033 \mathrm{E}-22$ \\
\hline 50 & $2.194 \mathrm{E}-18$ & $2.193 \mathrm{E}-18$ & $4.683 \mathrm{E}-21$ & $1.882 \mathrm{E}-21$ & $9.589 \mathrm{E}-23$ \\
\hline
\end{tabular}


b. Ritz chamber (cont’d).

\begin{tabular}{|c|c|c|c|c|c|}
\hline$E / k e V$ & $P_{\text {dep }}$ & $P_{\text {col }}$ & $S_{\text {col }}$ & $F_{\text {col }}$ & $G_{\text {col }}$ \\
\hline 52 & $2.113 \mathrm{E}-18$ & $2.112 \mathrm{E}-18$ & $4.470 \mathrm{E}-21$ & $1.669 \mathrm{E}-21$ & $8.974 \mathrm{E}-23$ \\
\hline 54 & $2.048 \mathrm{E}-18$ & $2.047 \mathrm{E}-18$ & $4.294 \mathrm{E}-21$ & $1.489 \mathrm{E}-21$ & $8.467 \mathrm{E}-23$ \\
\hline 56 & $1.998 \mathrm{E}-18$ & $1.995 \mathrm{E}-18$ & $4.148 \mathrm{E}-21$ & $1.338 \mathrm{E}-21$ & $8.052 \mathrm{E}-23$ \\
\hline 58 & $1.962 \mathrm{E}-18$ & $1.956 \mathrm{E}-18$ & $4.025 \mathrm{E}-21$ & $1.209 \mathrm{E}-21$ & $7.720 \mathrm{E}-23$ \\
\hline 60 & $1.938 \mathrm{E}-18$ & $1.927 \mathrm{E}-18$ & $3.920 \mathrm{E}-21$ & $1.098 \mathrm{E}-21$ & $7.458 \mathrm{E}-23$ \\
\hline 62 & $1.925 \mathrm{E}-18$ & $1.906 \mathrm{E}-18$ & $3.830 \mathrm{E}-21$ & $1.003 \mathrm{E}-21$ & $7.260 \mathrm{E}-23$ \\
\hline 64 & $1.922 \mathrm{E}-18$ & $1.893 \mathrm{E}-18$ & $3.752 \mathrm{E}-21$ & $9.198 \mathrm{E}-22$ & $7.118 \mathrm{E}-23$ \\
\hline 66 & $1.926 \mathrm{E}-18$ & $1.886 \mathrm{E}-18$ & $3.684 \mathrm{E}-21$ & $8.467 \mathrm{E}-22$ & $7.026 \mathrm{E}-23$ \\
\hline 68 & $1.938 \mathrm{E}-18$ & $1.884 \mathrm{E}-18$ & $3.625 \mathrm{E}-21$ & $7.817 \mathrm{E}-22$ & $6.979 \mathrm{E}-23$ \\
\hline 70 & $1.954 \mathrm{E}-18$ & $1.886 \mathrm{E}-18$ & $3.575 \mathrm{E}-21$ & $7.233 \mathrm{E}-22$ & $6.972 \mathrm{E}-23$ \\
\hline 72 & $1.974 \mathrm{E}-18$ & $1.891 \mathrm{E}-18$ & $3.535 \mathrm{E}-21$ & $6.702 \mathrm{E}-22$ & $7.001 \mathrm{E}-23$ \\
\hline 74 & $1.995 \mathrm{E}-18$ & $1.899 \mathrm{E}-18$ & $3.505 \mathrm{E}-21$ & $6.215 \mathrm{E}-22$ & $7.063 \mathrm{E}-23$ \\
\hline 76 & $2.018 \mathrm{E}-18$ & $1.910 \mathrm{E}-18$ & $3.487 \mathrm{E}-21$ & $5.765 \mathrm{E}-22$ & $7.154 \mathrm{E}-23$ \\
\hline 78 & $2.040 \mathrm{E}-18$ & $1.923 \mathrm{E}-18$ & $3.479 \mathrm{E}-21$ & $5.348 \mathrm{E}-22$ & $7.269 \mathrm{E}-23$ \\
\hline 80 & $2.063 \mathrm{E}-18$ & $1.938 \mathrm{E}-18$ & $3.481 \mathrm{E}-21$ & $4.961 \mathrm{E}-22$ & $7.405 \mathrm{E}-23$ \\
\hline 82 & $2.087 \mathrm{E}-18$ & $1.957 \mathrm{E}-18$ & $3.492 \mathrm{E}-21$ & $4.602 \mathrm{E}-22$ & $7.558 \mathrm{E}-23$ \\
\hline 84 & $2.114 \mathrm{E}-18$ & $1.980 \mathrm{E}-18$ & $3.508 \mathrm{E}-21$ & $4.271 \mathrm{E}-22$ & $7.723 \mathrm{E}-23$ \\
\hline 86 & $2.144 \mathrm{E}-18$ & $2.008 \mathrm{E}-18$ & $3.524 \mathrm{E}-21$ & $3.969 \mathrm{E}-22$ & $7.895 \mathrm{E}-23$ \\
\hline 88 & $2.180 \mathrm{E}-18$ & $2.041 \mathrm{E}-18$ & $3.536 \mathrm{E}-21$ & $3.696 \mathrm{E}-22$ & $8.070 \mathrm{E}-23$ \\
\hline 90 & $2.222 \mathrm{E}-18$ & $2.080 \mathrm{E}-18$ & $3.538 \mathrm{E}-21$ & $3.453 \mathrm{E}-22$ & $8.241 \mathrm{E}-23$ \\
\hline 92 & $2.270 \mathrm{E}-18$ & $2.125 \mathrm{E}-18$ & $3.530 \mathrm{E}-21$ & $3.240 \mathrm{E}-22$ & $8.403 \mathrm{E}-23$ \\
\hline 94 & $2.323 \mathrm{E}-18$ & $2.175 \mathrm{E}-18$ & $3.515 \mathrm{E}-21$ & $3.059 \mathrm{E}-22$ & $8.550 \mathrm{E}-23$ \\
\hline 96 & $2.376 \mathrm{E}-18$ & $2.227 \mathrm{E}-18$ & $3.506 \mathrm{E}-21$ & $2.907 \mathrm{E}-22$ & $8.675 \mathrm{E}-23$ \\
\hline 98 & $2.421 \mathrm{E}-18$ & $2.273 \mathrm{E}-18$ & $3.531 \mathrm{E}-21$ & $2.784 \mathrm{E}-22$ & $8.773 \mathrm{E}-23$ \\
\hline 100 & $2.450 \mathrm{E}-18$ & $2.306 \mathrm{E}-18$ & $3.641 \mathrm{E}-21$ & $2.687 \mathrm{E}-22$ & $8.838 \mathrm{E}-23$ \\
\hline
\end{tabular}


c. Wyckoff-Attix chamber.

\begin{tabular}{|c|c|c|c|c|c|}
\hline$E / \mathrm{keV}$ & $P_{\text {dep }}$ & $P_{\mathrm{col}}$ & $S_{\mathrm{col}}$ & $F_{\text {col }}$ & $G_{\mathrm{col}}$ \\
\hline 3.206 & $2.035 \mathrm{E}-16$ & $2.035 \mathrm{E}-16$ & $2.737 \mathrm{E}-18$ & $1.710 \mathrm{E}-18$ & $3.140 \mathrm{E}-21$ \\
\hline 5 & 1.414E-16 & 1.414E-16 & $1.691 \mathrm{E}-18$ & $1.073 \mathrm{E}-18$ & $2.601 \mathrm{E}-21$ \\
\hline 10 & $5.800 \mathrm{E}-17$ & $5.800 \mathrm{E}-17$ & 5.382E-19 & 3.383E-19 & 1.606E-21 \\
\hline 20 & $1.548 \mathrm{E}-17$ & $1.548 \mathrm{E}-17$ & $1.083 \mathrm{E}-19$ & 5.682E-20 & $7.284 \mathrm{E}-22$ \\
\hline 30 & $6.665 \mathrm{E}-18$ & $6.665 \mathrm{E}-18$ & $4.110 \mathrm{E}-20$ & 1.611E-20 & $4.041 E-22$ \\
\hline 40 & $4.041 \mathrm{E}-18$ & $4.041 \mathrm{E}-18$ & $2.322 \mathrm{E}-20$ & $6.548 \mathrm{E}-21$ & $2.656 \mathrm{E}-22$ \\
\hline 50 & $3.105 \mathrm{E}-18$ & $3.105 \mathrm{E}-18$ & $1.678 \mathrm{E}-20$ & $3.381 \mathrm{E}-21$ & $2.011 \mathrm{E}-22$ \\
\hline 60 & $2.794 \mathrm{E}-18$ & $2.794 \mathrm{E}-18$ & $1.408 \mathrm{E}-20$ & $2.039 \mathrm{E}-21$ & $1.708 \mathrm{E}-22$ \\
\hline 70 & $2.778 \mathrm{E}-18$ & $2.778 \mathrm{E}-18$ & 1.293E-20 & 1.355E-21 & $1.592 \mathrm{E}-22$ \\
\hline 80 & $2.928 \mathrm{E}-18$ & $2.928 \mathrm{E}-18$ & $1.254 \mathrm{E}-20$ & $9.573 \mathrm{E}-22$ & $1.595 \mathrm{E}-22$ \\
\hline 90 & $3.186 \mathrm{E}-18$ & 3.183E-18 & $1.256 \mathrm{E}-20$ & $7.035 \mathrm{E}-22$ & $1.688 \mathrm{E}-22$ \\
\hline 100 & $3.514 \mathrm{E}-18$ & $3.506 \mathrm{E}-18$ & 1.284E-20 & $5.319 \mathrm{E}-22$ & 1.859E-22 \\
\hline 110 & $3.889 \mathrm{E}-18$ & 3.873E-18 & $1.326 \mathrm{E}-20$ & $4.120 \mathrm{E}-22$ & $2.102 \mathrm{E}-22$ \\
\hline 120 & $4.296 \mathrm{E}-18$ & $4.271 \mathrm{E}-18$ & $1.378 \mathrm{E}-20$ & $3.269 \mathrm{E}-22$ & $2.416 \mathrm{E}-22$ \\
\hline 130 & $4.726 \mathrm{E}-18$ & 4.691E-18 & 1.436E-20 & $2.660 \mathrm{E}-22$ & $2.796 \mathrm{E}-22$ \\
\hline 140 & $5.172 \mathrm{E}-18$ & $5.128 \mathrm{E}-18$ & $1.496 \mathrm{E}-20$ & $2.223 \mathrm{E}-22$ & $3.236 \mathrm{E}-22$ \\
\hline 150 & $5.632 \mathrm{E}-18$ & 5.581E-18 & $1.556 \mathrm{E}-20$ & 1.910E-22 & $3.726 \mathrm{E}-22$ \\
\hline 160 & 6.104E-18 & 6.049E-18 & $1.616 \mathrm{E}-20$ & 1.686E-22 & $4.249 E-22$ \\
\hline 170 & $6.585 \mathrm{E}-18$ & $6.529 \mathrm{E}-18$ & $1.674 \mathrm{E}-20$ & 1.527E-22 & $4.790 \mathrm{E}-22$ \\
\hline 180 & 7.073E-18 & 7.019E-18 & 1.731E-20 & 1.414E-22 & $5.328 \mathrm{E}-22$ \\
\hline 190 & 7.564E-18 & 7.514E-18 & 1.784E-20 & 1.333E-22 & 5.847E-22 \\
\hline 200 & $8.056 \mathrm{E}-18$ & $8.011 \mathrm{E}-18$ & 1.833E-20 & 1.274E-22 & $6.332 \mathrm{E}-22$ \\
\hline 210 & $8.547 \mathrm{E}-18$ & 8.504E-18 & 1.876E-20 & 1.229E-22 & $6.778 \mathrm{E}-22$ \\
\hline 220 & $9.036 \mathrm{E}-18$ & 8.991E-18 & 1.910E-20 & 1.192E-22 & 7.182E-22 \\
\hline 230 & $9.527 \mathrm{E}-18$ & $9.470 \mathrm{E}-18$ & $1.936 \mathrm{E}-20$ & 1.158E-22 & $7.552 \mathrm{E}-22$ \\
\hline 240 & 1.002E-17 & $9.936 \mathrm{E}-18$ & $1.955 \mathrm{E}-20$ & 1.127E-22 & $7.900 \mathrm{E}-22$ \\
\hline 250 & $1.052 \mathrm{E}-17$ & $1.038 \mathrm{E}-17$ & 1.971E-20 & 1.095E-22 & 8.241E-22 \\
\hline 260 & 1.102E-17 & $1.080 \mathrm{E}-17$ & 1.991E-20 & 1.061E-22 & 8.597E-22 \\
\hline 270 & 1.152E-17 & $1.116 \mathrm{E}-17$ & 2.019E-20 & $1.024 \mathrm{E}-22$ & 8.990E-22 \\
\hline 280 & $1.201 \mathrm{E}-17$ & $1.146 \mathrm{E}-17$ & $2.054 \mathrm{E}-20$ & $9.788 \mathrm{E}-23$ & $9.443 \mathrm{E}-22$ \\
\hline 290 & $1.248 \mathrm{E}-17$ & 1.169E-17 & 2.074E-20 & $9.169 \mathrm{E}-23$ & $9.978 \mathrm{E}-22$ \\
\hline 300 & $1.297 \mathrm{E}-17$ & $1.190 \mathrm{E}-17$ & $2.017 \mathrm{E}-20$ & $8.262 \mathrm{E}-23$ & $1.062 \mathrm{E}-21$ \\
\hline
\end{tabular}


Table 6. The monoenergetic correction factors reconstructed form the adopted, smoothed data from Burns' Monte Carlo calculations for NIST FACs. Values for $k_{\mathrm{fl}}$ at energies below $3.206 \mathrm{keV}$ are not given, as they are not expected to have any meaning. The large number of significant figures is for consistency with Table 3.

a. Lamperti chamber.

\begin{tabular}{|c|c|c|c|c|}
\hline$E / \mathrm{keV}$ & $k_{\mathrm{el}}$ & $k_{\mathrm{SC}}$ & $k_{\mathrm{fl}}$ & $k_{\mathrm{br}}$ \\
\hline 2 & 1.0000000 & 0.9954059 & & 0.9999979 \\
\hline 4 & 1.0000000 & 0.9969391 & 0.9943817 & 0.9999967 \\
\hline 6 & 1.0000000 & 0.9976498 & 0.9956150 & 0.9999948 \\
\hline 8 & 1.0000000 & 0.9980605 & 0.9963734 & 0.9999921 \\
\hline 10 & 1.0000000 & 0.9983340 & 0.9969165 & 0.9999887 \\
\hline 12 & 1.0000000 & 0.9985289 & 0.9973341 & 0.9999848 \\
\hline 14 & 1.0000000 & 0.9986697 & 0.9976620 & 0.9999808 \\
\hline 16 & 1.0000000 & 0.9987698 & 0.9979203 & 0.9999768 \\
\hline 18 & 1.0000000 & 0.9988387 & 0.9981247 & 0.9999731 \\
\hline 20 & 1.0000000 & 0.9988842 & 0.9982890 & 0.9999699 \\
\hline 22 & 1.0000000 & 0.9989130 & 0.9984247 & 0.9999672 \\
\hline 24 & 1.0000000 & 0.9989308 & 0.9985409 & 0.9999651 \\
\hline 26 & 1.0000000 & 0.9989417 & 0.9986442 & 0.9999638 \\
\hline 28 & 1.0000000 & 0.9989490 & 0.9987385 & 0.9999630 \\
\hline 30 & 1.0000000 & 0.9989546 & 0.9988258 & 0.9999627 \\
\hline 32 & 1.0000000 & 0.9989599 & 0.9989075 & 0.9999629 \\
\hline 34 & 1.0001842 & 0.9989658 & 0.9989840 & 0.9999632 \\
\hline 36 & 1.0018207 & 0.9989722 & 0.9990552 & 0.9999636 \\
\hline 38 & 1.0058194 & 0.9989797 & 0.9991215 & 0.9999640 \\
\hline 40 & 1.0135379 & 0.9989884 & 0.9991829 & 0.9999643 \\
\hline 42 & 1.0262898 & 0.9989968 & 0.9992386 & 0.9999644 \\
\hline 44 & 1.0451052 & 0.9990013 & 0.9992863 & 0.9999640 \\
\hline 46 & 1.0698429 & 0.9989933 & 0.9993213 & 0.9999629 \\
\hline 48 & 1.0983734 & 0.9989571 & 0.9993353 & 0.9999604 \\
\hline 50 & 1.1251489 & 0.9988627 & 0.9993131 & 0.9999550 \\
\hline & & & &
\end{tabular}


b. Ritz chamber.

\begin{tabular}{|c|c|c|c|c|}
\hline$E / \mathrm{keV}$ & $k_{\mathrm{el}}$ & $k_{\mathrm{sc}}$ & $k_{\mathrm{fl}}$ & $k_{\mathrm{br}}$ \\
\hline 2 & 1.0000000 & 0.9916142 & & 0.9999973 \\
\hline 4 & 1.0000000 & 0.9934071 & 0.9916391 & 0.9999956 \\
\hline 6 & 1.0000000 & 0.9946713 & 0.9934059 & 0.9999930 \\
\hline 8 & 1.0000000 & 0.9955520 & 0.9945676 & 0.9999894 \\
\hline 10 & 1.0000000 & 0.9961639 & 0.9953799 & 0.9999849 \\
\hline 12 & 1.0000000 & 0.9965896 & 0.9959779 & 0.9999797 \\
\hline 14 & 1.0000000 & 0.9968865 & 0.9964369 & 0.9999740 \\
\hline 16 & 1.0000000 & 0.9970941 & 0.9968014 & 0.9999682 \\
\hline 18 & 1.0000000 & 0.9972401 & 0.9970991 & 0.9999628 \\
\hline 20 & 1.0000000 & 0.9973435 & 0.9973475 & 0.9999580 \\
\hline 22 & 1.0000000 & 0.9974186 & 0.9975589 & 0.9999540 \\
\hline 24 & 1.0000000 & 0.9974751 & 0.9977420 & 0.9999509 \\
\hline 26 & 1.0000000 & 0.9975197 & 0.9979031 & 0.9999487 \\
\hline 28 & 1.0000000 & 0.9975573 & 0.9980472 & 0.9999473 \\
\hline 30 & 1.0000000 & 0.9975907 & 0.9981782 & 0.9999466 \\
\hline 32 & 1.0000000 & 0.9976218 & 0.9982988 & 0.9999464 \\
\hline 34 & 1.0000000 & 0.9976513 & 0.9984115 & 0.9999467 \\
\hline 36 & 1.0000000 & 0.9976796 & 0.9985178 & 0.9999473 \\
\hline 38 & 1.0000000 & 0.9977066 & 0.9986190 & 0.9999482 \\
\hline 40 & 1.0000000 & 0.9977323 & 0.9987157 & 0.9999493 \\
\hline 42 & 1.0000000 & 0.9977568 & 0.9988082 & 0.9999505 \\
\hline 44 & 1.0000000 & 0.9977801 & 0.9988967 & 0.9999518 \\
\hline 46 & 1.0000000 & 0.9978027 & 0.9989810 & 0.9999532 \\
\hline 48 & 1.0000000 & 0.9978251 & 0.9990608 & 0.9999546 \\
\hline 50 & 1.0001842 & 0.9978693 & 0.9991445 & 0.9999564 \\
\hline & & & &
\end{tabular}


b. Ritz chamber (cont'd).

\begin{tabular}{|c|c|c|c|c|}
\hline$E / \mathrm{keV}$ & $k_{\mathrm{el}}$ & $k_{\mathrm{sc}}$ & $k_{\mathrm{fl}}$ & $k_{\mathrm{br}}$ \\
\hline 52 & 1.0003224 & 0.9978882 & 0.9992122 & 0.9999576 \\
\hline 54 & 1.0006910 & 0.9979065 & 0.9992744 & 0.9999588 \\
\hline 56 & 1.0014978 & 0.9979257 & 0.9993314 & 0.9999598 \\
\hline 58 & 1.0031364 & 0.9979465 & 0.9993837 & 0.9999606 \\
\hline 60 & 1.0058425 & 0.9979695 & 0.9994315 & 0.9999614 \\
\hline 62 & 1.0098340 & 0.9979947 & 0.9994753 & 0.9999620 \\
\hline 64 & 1.0151261 & 0.9980219 & 0.9995153 & 0.9999625 \\
\hline 66 & 1.0215274 & 0.9980505 & 0.9995521 & 0.9999628 \\
\hline 68 & 1.0287504 & 0.9980795 & 0.9995860 & 0.9999630 \\
\hline 70 & 1.0363823 & 0.9981077 & 0.9996173 & 0.9999631 \\
\hline 72 & 1.0439026 & 0.9981340 & 0.9996463 & 0.9999631 \\
\hline 74 & 1.0507998 & 0.9981575 & 0.9996734 & 0.9999629 \\
\hline 76 & 1.0566715 & 0.9981775 & 0.9996987 & 0.9999626 \\
\hline 78 & 1.0612800 & 0.9981938 & 0.9997224 & 0.9999623 \\
\hline 80 & 1.0645351 & 0.9982071 & 0.9997446 & 0.9999619 \\
\hline 82 & 1.0665470 & 0.9982190 & 0.9997654 & 0.9999615 \\
\hline 84 & 1.0675789 & 0.9982316 & 0.9997847 & 0.9999611 \\
\hline 86 & 1.0679969 & 0.9982478 & 0.9998027 & 0.9999608 \\
\hline 88 & 1.0681445 & 0.9982705 & 0.9998192 & 0.9999605 \\
\hline 90 & 1.0681937 & 0.9983018 & 0.9998343 & 0.9999605 \\
\hline 92 & 1.0682183 & 0.9983417 & 0.9998478 & 0.9999605 \\
\hline 94 & 1.0679231 & 0.9983867 & 0.9998596 & 0.9999608 \\
\hline 96 & 1.0669646 & 0.9984280 & 0.9998697 & 0.9999611 \\
\hline 98 & 1.0650010 & 0.9984495 & 0.9998777 & 0.9999615 \\
\hline 100 & 1.0623803 & 0.9984232 & 0.9998837 & 0.9999617 \\
\hline
\end{tabular}


c. Wyckoff-Attix chamber.

\begin{tabular}{|c|c|c|c|c|}
\hline$E / \mathrm{keV}$ & $k_{\mathrm{el}}$ & $k_{\mathrm{sc}}$ & $k_{\mathrm{fl}}$ & $k_{\mathrm{br}}$ \\
\hline 3.206 & 1.0000000 & 0.9867256 & 0.9917779 & 0.9999849 \\
\hline 5 & 1.0000000 & 0.9881827 & 0.9925562 & 0.9999820 \\
\hline 10 & 1.0000000 & 0.9908061 & 0.9942537 & 0.9999727 \\
\hline 20 & 1.0000000 & 0.9930521 & 0.9963685 & 0.9999534 \\
\hline 30 & 1.0000000 & 0.9938706 & 0.9976026 & 0.9999399 \\
\hline 40 & 1.0000000 & 0.9942853 & 0.9983913 & 0.9999347 \\
\hline 50 & 1.0000000 & 0.9946267 & 0.9989182 & 0.9999357 \\
\hline 60 & 1.0000000 & 0.9949880 & 0.9992746 & 0.9999392 \\
\hline 70 & 1.0000000 & 0.9953677 & 0.9995147 & 0.9999430 \\
\hline 80 & 1.0000000 & 0.9957372 & 0.9996746 & 0.9999458 \\
\hline 90 & 1.0006910 & 0.9960689 & 0.9997799 & 0.9999472 \\
\hline 100 & 1.0022591 & 0.9963514 & 0.9998488 & 0.9999472 \\
\hline 110 & 1.0040839 & 0.9965870 & 0.9998940 & 0.9999459 \\
\hline 120 & 1.0059584 & 0.9967829 & 0.9999237 & 0.9999436 \\
\hline 130 & 1.0075347 & 0.9969484 & 0.9999435 & 0.9999406 \\
\hline 140 & 1.0086489 & 0.9970918 & 0.9999568 & 0.9999371 \\
\hline 150 & 1.0091832 & 0.9972195 & 0.9999659 & 0.9999334 \\
\hline 160 & 1.0091135 & 0.9973355 & 0.9999722 & 0.9999299 \\
\hline 170 & 1.0085792 & 0.9974420 & 0.9999767 & 0.9999268 \\
\hline 180 & 1.0076971 & 0.9975401 & 0.9999799 & 0.9999243 \\
\hline 190 & 1.0066767 & 0.9976312 & 0.9999823 & 0.9999224 \\
\hline 200 & 1.0057036 & 0.9977169 & 0.9999841 & 0.9999211 \\
\hline 210 & 1.0050785 & 0.9977993 & 0.9999856 & 0.9999205 \\
\hline 220 & 1.0050323 & 0.9978800 & 0.9999868 & 0.9999203 \\
\hline 230 & 1.0059815 & 0.9979596 & 0.9999878 & 0.9999204 \\
\hline 240 & 1.0084399 & 0.9980361 & 0.9999887 & 0.9999207 \\
\hline 250 & 1.0130479 & 0.9981051 & 0.9999895 & 0.9999208 \\
\hline 260 & 1.0206339 & 0.9981597 & 0.9999902 & 0.9999205 \\
\hline 270 & 1.0320720 & 0.9981945 & 0.9999908 & 0.9999196 \\
\hline 280 & 1.0478521 & 0.9982107 & 0.9999915 & 0.9999177 \\
\hline 290 & 1.0677756 & 0.9982288 & 0.9999922 & 0.9999148 \\
\hline 300 & 1.0898080 & 0.9983084 & 0.9999931 & 0.9999110 \\
\hline & & & & \\
\hline
\end{tabular}


Table 7. Estimated relative standard uncertainties due to smoothing of the Burns data adopted for the NIST FACs.

\begin{tabular}{|c|c|c|c|}
\hline $\begin{array}{c}\text { Correction } \\
\text { Factor }\end{array}$ & $\begin{array}{c}\text { Lamperti } \\
(10 \mathrm{kV}-50 \mathrm{kV})\end{array}$ & $\begin{array}{c}\text { Ritz } \\
(20 \mathrm{kV}-100 \mathrm{kV})\end{array}$ & $\begin{array}{c}\text { Wyckoff-Attix } \\
(50 \mathrm{kV}-300 \mathrm{kV})\end{array}$ \\
\hline$k_{\mathrm{el}}$ & $0.02 \%$ & $0.06 \%$ & $0.04 \%$ \\
\hline$k_{\mathrm{sc}}$ & $0.01 \%$ & $0.01 \%$ & $0.01 \%$ \\
\hline$k_{\mathrm{fl}}$ & $0.01 \%$ & $0.01 \%$ & $0.01 \%$ \\
\hline
\end{tabular}

Table 8. Estimated relative standard uncertainties inherent in the data adopted for calculating correction factors for the NIST FACs (combining results in Tables 2 and 7). The last row gives the estimate associated with the assumption of $k_{\mathrm{br}} /(1-$ $\bar{g})=1$.

\begin{tabular}{|c|c|c|c|}
\hline $\begin{array}{c}\text { Correction } \\
\text { Factor }\end{array}$ & $\begin{array}{c}\text { Lamperti } \\
(10 \mathrm{kV}-50 \mathrm{kV})\end{array}$ & $\begin{array}{c}\text { Ritz } \\
(20 \mathrm{kV}-100 \mathrm{kV})\end{array}$ & $\begin{array}{c}\text { Wyckoff-Attix } \\
(50 \mathrm{kV}-300 \mathrm{kV})\end{array}$ \\
\hline$k_{\mathrm{el}}$ & $0.05 \%$ & $0.08 \%$ & $0.10 \%$ \\
\hline$k_{\mathrm{sc}}$ & $0.03 \%$ & $0.03 \%$ & $0.03 \%$ \\
\hline$k_{\mathrm{fl}}$ & $0.05 \%$ & $0.05 \%$ & $0.03 \%$ \\
\hline$k_{\mathrm{br}} /(1-\mathrm{g})$ & $0.02 \%$ & $0.02 \%$ & $0.03 \%$ \\
\hline
\end{tabular}


Table 9. Adopted correction factors for the NIST free-air chambers: traditional NIST beam qualities.

\begin{tabular}{|c|c|c|c|c|c|c|c|c|c|}
\hline \multirow{2}{*}{$\begin{array}{l}\text { Beam } \\
\text { Code }\end{array}$} & \multicolumn{3}{|c|}{ Lamperti } & \multicolumn{3}{|c|}{ Ritz } & \multicolumn{3}{|c|}{ Wyckoff-Attix } \\
\hline & $k_{\mathrm{el}}$ & $k_{\mathrm{SC}}$ & $k_{\mathrm{fl}}$ & $k_{\mathrm{el}}$ & $k_{\mathrm{SC}}$ & $k_{\mathrm{fl}}$ & $k_{\mathrm{el}}$ & $k_{\mathrm{SC}}$ & $k_{\mathrm{fl}}$ \\
\hline L10 & 1.00000 & 0.99790 & 0.99608 & & & & & & \\
\hline L15 & 1.00000 & 0.99818 & 0.99662 & & & & & & \\
\hline L20 & 1.00000 & 0.99835 & 0.99698 & 1.00000 & 0.99622 & 0.99549 & & & \\
\hline L30 & 1.00000 & 0.99860 & 0.99758 & 1.00000 & 0.99676 & 0.99635 & & & \\
\hline L40 & 1.00000 & 0.99874 & 0.99798 & 1.00000 & 0.99707 & 0.99694 & & & \\
\hline L50 & 1.00153 & 0.99884 & 0.99831 & 1.00000 & 0.99730 & 0.99740 & 1.00000 & 0.99308 & 0.99656 \\
\hline L80 & & & & 1.00128 & 0.99756 & 0.99810 & 1.00000 & 0.99377 & 0.99750 \\
\hline L100 & & & & 1.00531 & 0.99764 & 0.99838 & 1.00002 & 0.99407 & 0.99792 \\
\hline M20 & 1.00000 & 0.99851 & 0.99732 & 1.00000 & 0.99656 & 0.99598 & & & \\
\hline M30 & 1.00000 & 0.99874 & 0.99790 & 1.00000 & 0.99704 & 0.99681 & & & \\
\hline M40 & 1.00008 & 0.99883 & 0.99823 & 1.00000 & 0.99726 & 0.99729 & & & \\
\hline M50 & 1.00202 & 0.99888 & 0.99844 & 1.00000 & 0.99738 & 0.99760 & 1.00000 & 0.99328 & 0.99681 \\
\hline M60 & & & & 1.00003 & 0.99752 & 0.99796 & 1.00000 & 0.99363 & 0.99730 \\
\hline M80 & & & & 1.00487 & 0.99766 & 0.99844 & 1.00000 & 0.99409 & 0.99796 \\
\hline M100 & & & & 1.00946 & 0.99780 & 0.99885 & 1.00003 & 0.99453 & 0.99855 \\
\hline M120 & & & & & & & 1.00034 & 0.99487 & 0.99889 \\
\hline M150 & & & & & & & 1.00133 & 0.99548 & 0.99937 \\
\hline M200 & & & & & & & 1.00414 & 0.99637 & 0.99975 \\
\hline M250 & & & & & & & 1.00628 & 0.99712 & 0.99993 \\
\hline M300 & & & & & & & 1.01227 & 0.99780 & 0.99998 \\
\hline & & & & & & & & & \\
\hline $\mathrm{H} 10$ & 1.00000 & 0.99801 & 0.99629 & & & & & & \\
\hline $\mathrm{H} 15$ & 1.00000 & 0.99847 & 0.99720 & & & & & & \\
\hline $\mathrm{H} 2 \mathrm{O}$ & 1.00000 & 0.99871 & 0.99777 & 1.00000 & 0.99696 & 0.99661 & & & \\
\hline $\mathrm{H} 30$ & 1.00000 & 0.99892 & 0.99849 & 1.00000 & 0.99744 & 0.99766 & & & \\
\hline $\mathrm{H} 40$ & 1.00089 & 0.99896 & 0.99889 & 1.00000 & 0.99762 & 0.99828 & & & \\
\hline $\mathrm{H} 50$ & 1.01727 & 0.99898 & 0.99909 & 1.00000 & 0.99770 & 0.99859 & 1.00000 & 0.99419 & 0.99820 \\
\hline $\mathrm{H} 60$ & & & & 1.00035 & 0.99781 & 0.99899 & 1.00000 & 0.99452 & 0.99873 \\
\hline $\mathrm{H} 100$ & & & & 1.05982 & 0.99824 & 0.99976 & 1.00045 & 0.99584 & 0.99970 \\
\hline $\mathrm{H} 150$ & & & & & & & 1.00575 & 0.99674 & 0.99991 \\
\hline $\mathrm{H} 200$ & & & & & & & 1.00815 & 0.99737 & 0.99997 \\
\hline $\mathrm{H} 250$ & & & & & & & 1.00633 & 0.99777 & 0.99998 \\
\hline H300 & & & & & & & 1.02266 & 0.99808 & 0.99999 \\
\hline & & & & & & & & & \\
\hline S60 & & & & 1.00005 & 0.99763 & 0.99835 & 1.00000 & 0.99399 & 0.99784 \\
\hline S75 & & & & 1.00080 & 0.99755 & 0.99810 & 1.00000 & 0.99376 & 0.99750 \\
\hline
\end{tabular}


Table 10. Adopted correction factors for the NIST free-air chambers: ISO beam qualities. The NS10, NS15, NS20, NS30, and NS40 beam qualities are assumed to be the same as that of the NIST H10, H15, H20, H30, and H40 beams, respectively.

\begin{tabular}{|c|c|c|c|c|c|c|c|c|c|}
\hline \multirow{2}{*}{$\begin{array}{l}\text { Beam } \\
\text { Code }\end{array}$} & \multicolumn{3}{|c|}{ Lamperti } & \multicolumn{3}{|c|}{ Ritz } & \multicolumn{3}{|c|}{ Wyckoff-Attix } \\
\hline & $k_{\mathrm{el}}$ & $k_{\mathrm{sC}}$ & $k_{\mathrm{fl}}$ & $k_{\mathrm{el}}$ & $k_{\mathrm{sC}}$ & $k_{\mathrm{fl}}$ & $k_{\mathrm{el}}$ & $k_{\mathrm{sC}}$ & $k_{\mathrm{fl}}$ \\
\hline HK10 & 1.00000 & 0.99790 & 0.99608 & & & & & & \\
\hline HK20 & 1.00000 & 0.99841 & 0.99712 & 1.00000 & 0.99636 & 0.99568 & & & \\
\hline HK30 & 1.00000 & 0.99872 & 0.99786 & 1.00000 & 0.99700 & 0.99675 & & & \\
\hline HK60 & & & & 1.00005 & 0.99760 & 0.99824 & 1.00000 & 0.99389 & 0.99769 \\
\hline HK100 & & & & 1.01306 & 0.99789 & 0.99910 & 1.00004 & 0.99478 & 0.99888 \\
\hline HK200 & & & & & & & 1.00414 & 0.99637 & 0.99975 \\
\hline HK250 & & & & & & & 1.00541 & 0.99687 & 0.99987 \\
\hline HK280 & & & & & & & 1.00706 & 0.99724 & 0.99994 \\
\hline HK300 & & & & & & & 1.00854 & 0.99727 & 0.99993 \\
\hline WS60 & & & & 1.00020 & 099776 & 0.99881 & 1.00000 & 099438 & 0.99850 \\
\hline WS80 & & & & 1.00958 & 0.99792 & 0.99923 & 1.00000 & 0.99482 & 0.99904 \\
\hline WS110 & & & & & & & 1.00058 & 0.99570 & 0.99961 \\
\hline WS150 & & & & & & & 1.00400 & 0.99646 & 0.99984 \\
\hline WS200 & & & & & & & 1.00722 & 0.99708 & 0.99994 \\
\hline WS250 & & & & & & & 1.00716 & 0.99751 & 0.99997 \\
\hline WS300 & & & & & & & 1.01227 & 0.99780 & 0.99998 \\
\hline & & & & & & & & & \\
\hline NS10 & 1.00000 & 0.99801 & 0.99629 & & & & & & \\
\hline NS15 & 1.00000 & 0.99847 & 0.99720 & & & & & & \\
\hline NS20 & 1.00000 & 0.99871 & 0.99777 & 1.00000 & 0.99696 & 0.99661 & & & \\
\hline NS25 & 1.00000 & 0.99884 & 0.99815 & 1.00000 & 0.99724 & 0.99715 & & & \\
\hline NS30 & 1.00000 & 0.99892 & 0.99849 & 1.00000 & 0.99744 & 0.99766 & & & \\
\hline NS40 & 1.00089 & 0.99896 & 0.99889 & 1.00000 & 0.99762 & 0.99828 & & & \\
\hline NS60 & & & & 1.00035 & 0.99781 & 0.99899 & 1.00000 & 0.99452 & 0.99873 \\
\hline NS80 & & & & 1.02295 & 0.99804 & 0.99951 & 1.00000 & 0.99517 & 0.99937 \\
\hline NS100 & & & & 1.05982 & 0.99824 & 0.99976 & 1.00045 & 0.99584 & 0.99970 \\
\hline NS120 & & & & & & & 1.00260 & 0.99635 & 0.99984 \\
\hline NS150 & & & & & & & 1.00569 & 0.99674 & 0.99991 \\
\hline NS200 & & & & & & & 1.00814 & 0.99739 & 0.99997 \\
\hline NS250 & & & & & & & 1.00634 & 0.99779 & 0.99998 \\
\hline NS300 & & & & & & & 1.02266 & 0.99808 & 0.99999 \\
\hline $1 K 10$ & & & $0006 \wedge 6$ & & & & & & \\
\hline LKIO & 1.00000 & 0.99810 & 0.99646 & & & & & & \\
\hline LK20 & 1.00000 & 0.99878 & 0.99795 & 1.00000 & 0.99711 & 0.99685 & & & \\
\hline LK30 & 1.00000 & 0.99894 & 0.99860 & 1.00000 & 0.99750 & 0.99784 & & & \\
\hline LK35 & 1.00002 & 0.99895 & 0.99880 & 1.00000 & 0.99758 & 0.99813 & & & \\
\hline LK55 & & & & 1.00012 & 0.99782 & 0.99901 & 1.00000 & 0.99452 & 0.99876 \\
\hline LK70 & & & & 1.01031 & 0.99798 & 0.99942 & 1.00000 & 0.99501 & 0.99927 \\
\hline LK100 & & & & 1.06452 & 0.99828 & 0.99980 & 1.00064 & 0.99596 & 0.99974 \\
\hline LK125 & & & & & & & 1.00405 & 0.99656 & 0.99989 \\
\hline LK170 & & & & & & & 1.00868 & 0.99719 & 0.99996 \\
\hline LK210 & & & & & & & 1.00703 & 0.99758 & 0.99998 \\
\hline LK240 & & & & & & & 1.00575 & 0.99782 & 0.99999 \\
\hline
\end{tabular}


Table 11. Adopted correction factors for the NIST free-air chambers: BIPM/CCRI beam qualities. The BIPM10 beam quality is assumed to be the same as that of the ISO HK10 beam; the BIPM50b beam quality is assumed to be the same as that of the NIST M50 beam.

\begin{tabular}{|l|c|c|c|c|c|c|c|c|c|}
\hline \multirow{2}{*}{$\begin{array}{l}\text { Beam } \\
\text { Code }\end{array}$} & \multicolumn{3}{|c|}{ Lamperti } & \multicolumn{3}{c|}{ Ritz } & \multicolumn{3}{c|}{ Wyckoff-Attix } \\
\cline { 2 - 10 } & $k_{\mathrm{el}}$ & $k_{\mathrm{sc}}$ & $k_{\mathrm{fl}}$ & $k_{\mathrm{el}}$ & $k_{\mathrm{sc}}$ & $k_{\mathrm{fl}}$ & $k_{\mathrm{el}}$ & $k_{\mathrm{sc}}$ & $k_{\mathrm{fl}}$ \\
\hline BIPM10 & 1.00000 & 0.99790 & 0.99608 & 1.00000 & 0.99523 & 0.99415 & & & \\
\hline BIPM25 & 1.00000 & 0.99858 & 0.99751 & 1.00000 & 0.99672 & 0.99625 & & & \\
\hline BIPM30 & 1.00000 & 0.99790 & 0.99608 & 1.00000 & 0.99523 & 0.99415 & & & \\
\hline BIPM50A & 1.00530 & 0.99895 & 0.99883 & 1.00000 & 0.99749 & 0.99788 & & & \\
\hline BIPM50B & 1.00202 & 0.99888 & 0.99844 & 1.00000 & 0.99738 & 0.99760 & 1.00000 & 0.99328 & 0.99681 \\
\hline & & & & & & & & & \\
\hline BIPM100 & & & & 1.00709 & 0.99773 & 0.99862 & 1.00002 & 0.99431 & 0.99824 \\
\hline BIPM135 & & & & & & & 1.00068 & 0.99520 & 0.99918 \\
\hline BIPM180 & & & & & & & 1.00281 & 0.99588 & 0.99951 \\
\hline BIPM250 & & & & & & & 1.00556 & 0.99691 & 0.99986 \\
\hline
\end{tabular}




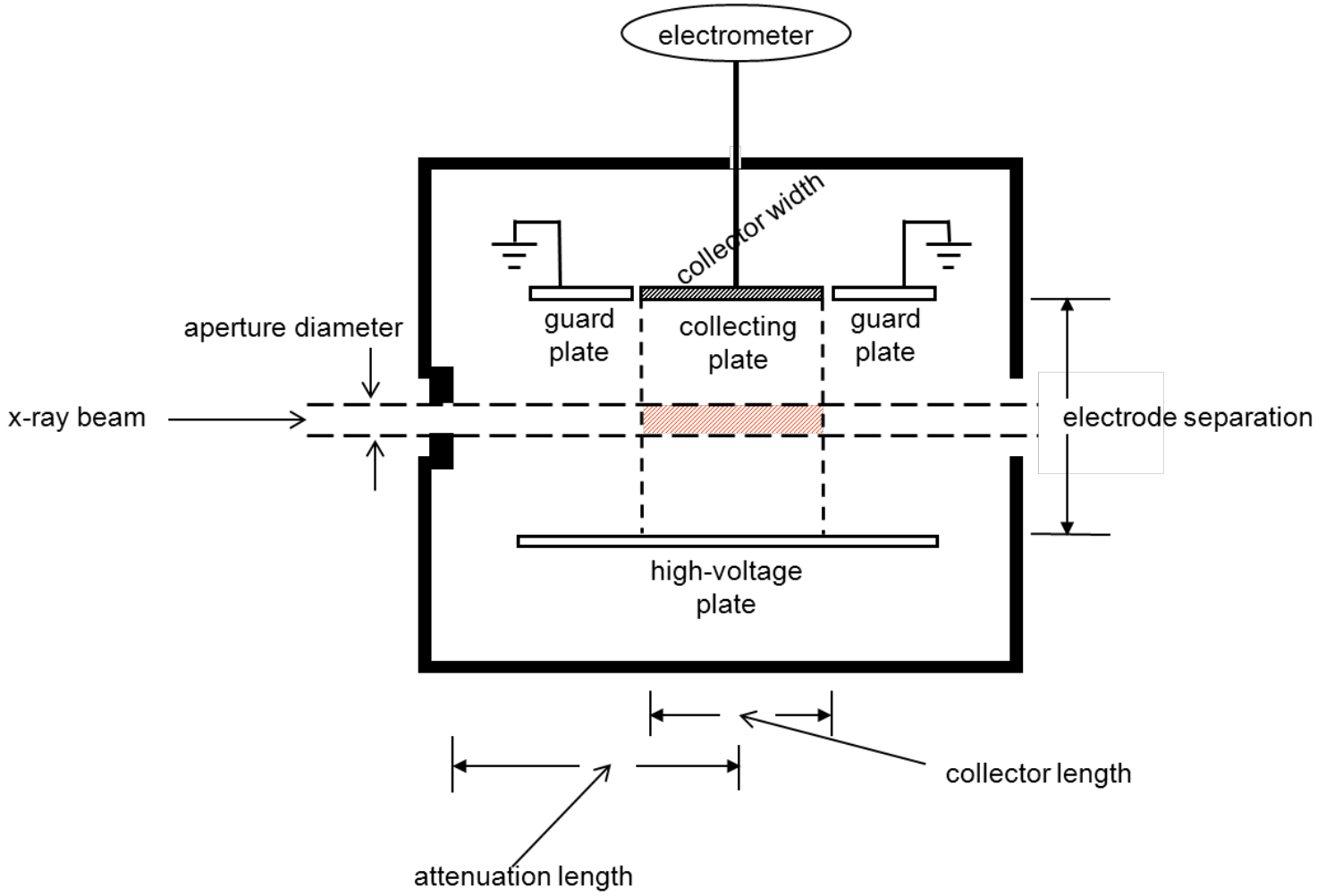

Figure 1. Schematic of a parallel-plate free-air ionization chamber used by the NIST for the realization of air kerma for $\mathrm{W}$-anode $\mathrm{x}$-ray beams. The high-voltage, guard, and collecting plates have a width that extends into the paper. 


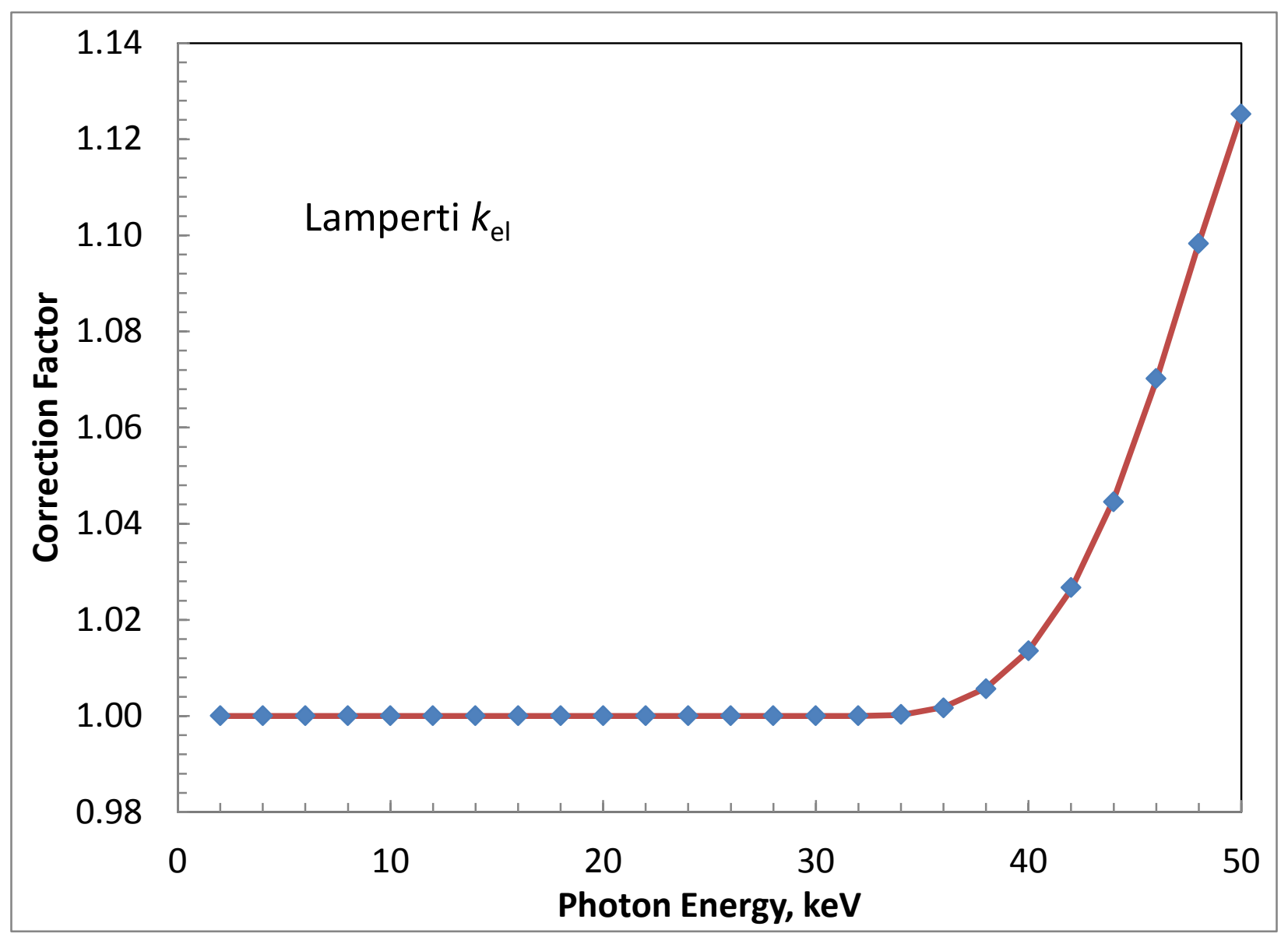

Figure 2. Comparison of original Burns data (points) for the Lamperti free-air chamber with the results from the smoothed underlying data (curve).

a. Correction factor $k_{\mathrm{el}}$. 


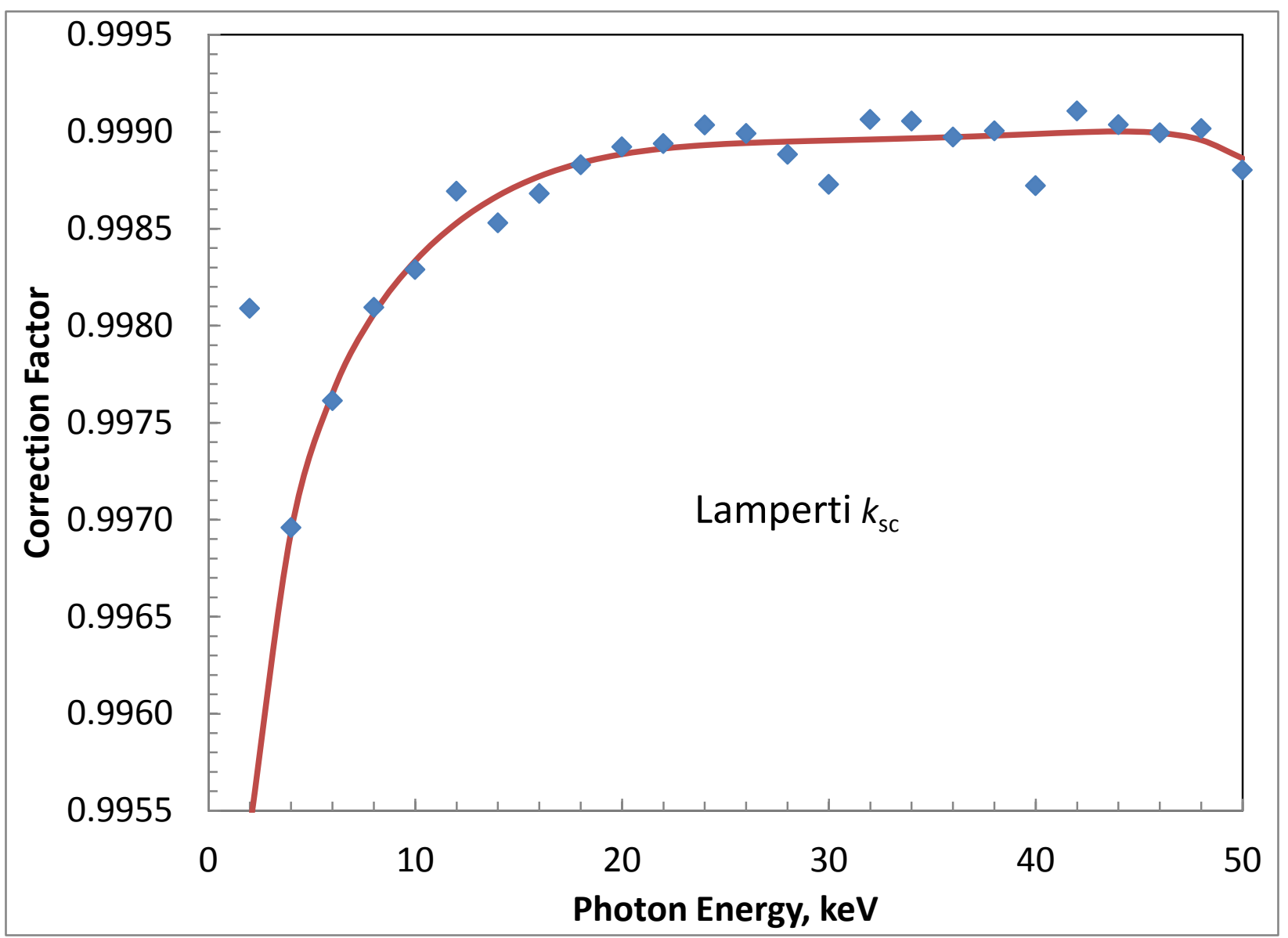

Figure 2. Comparison of original Burns data (points) for the Lamperti free-air chamber with the results from the smoothed underlying data (curve).

b. Correction factor $k_{\mathrm{sc}}$. 


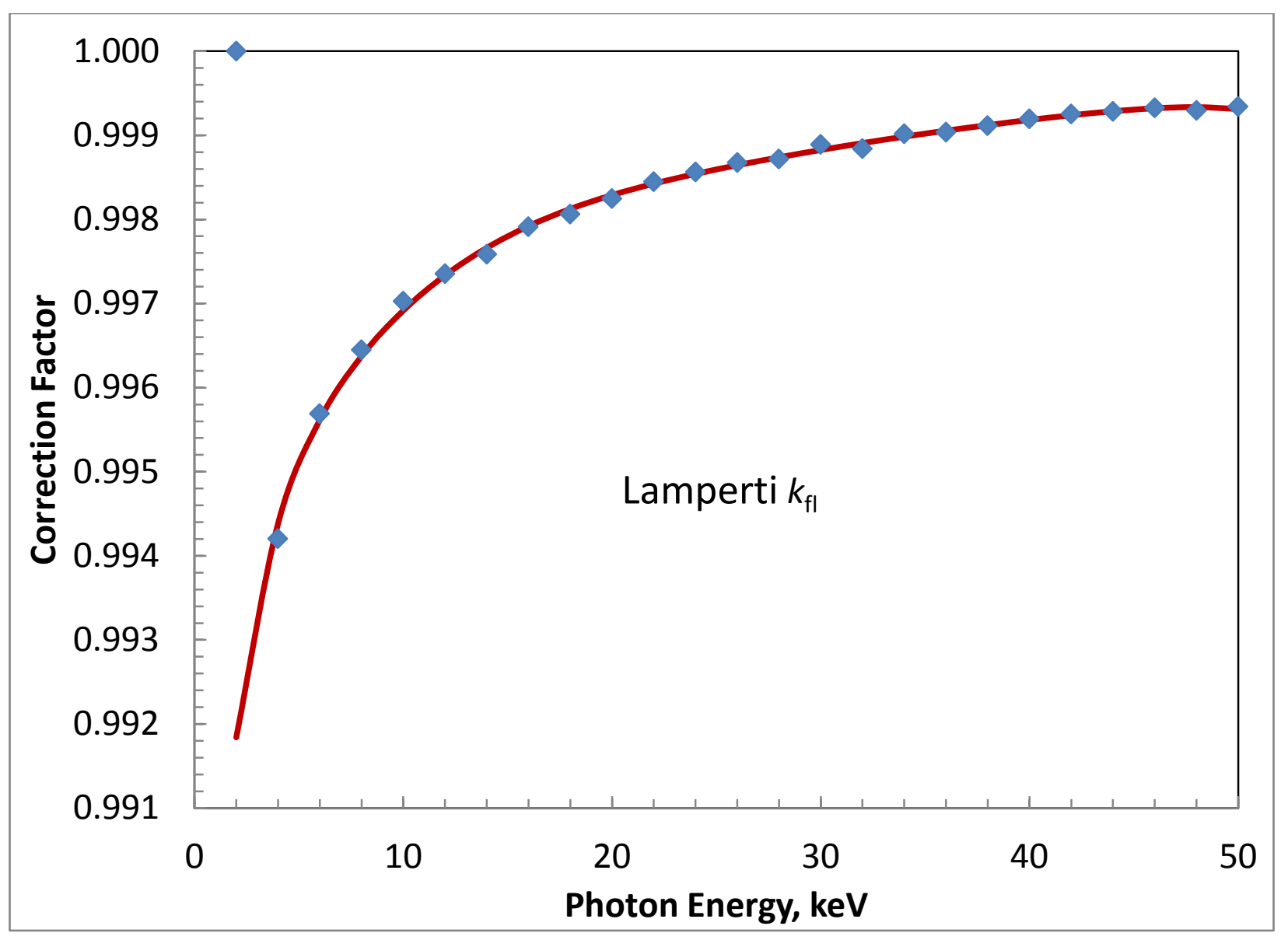

Figure 2. Comparison of original Burns data (points) for the Lamperti free-air chamber with the results from the smoothed underlying data (curve).

c. Correction factor $k_{\mathrm{fl}}$. 


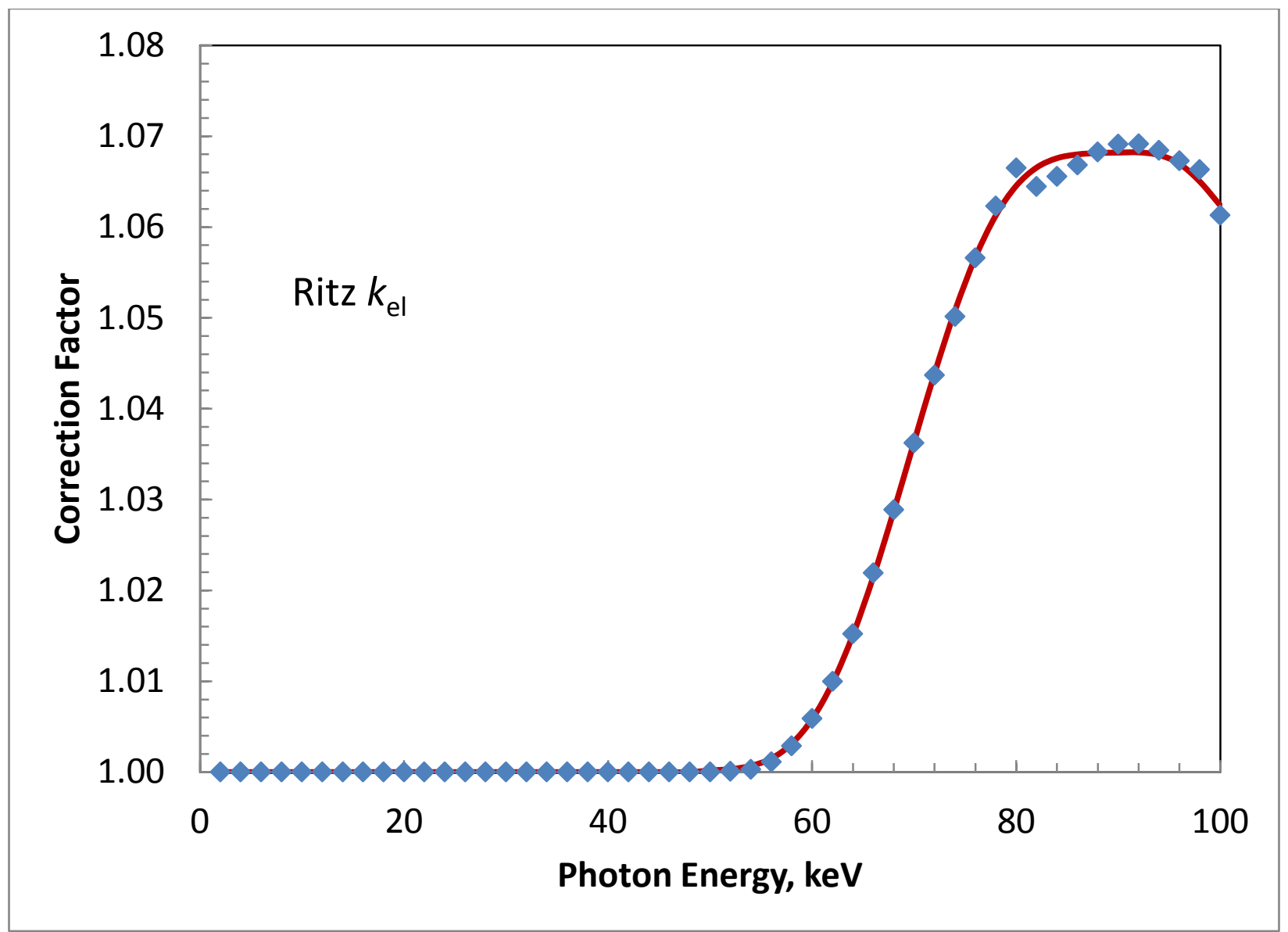

Figure 3. Comparison of original Burns data (points) for the Ritz free-air chamber with the results from the smoothed underlying data (curve).

a. Correction factor $k_{\mathrm{el}}$. 


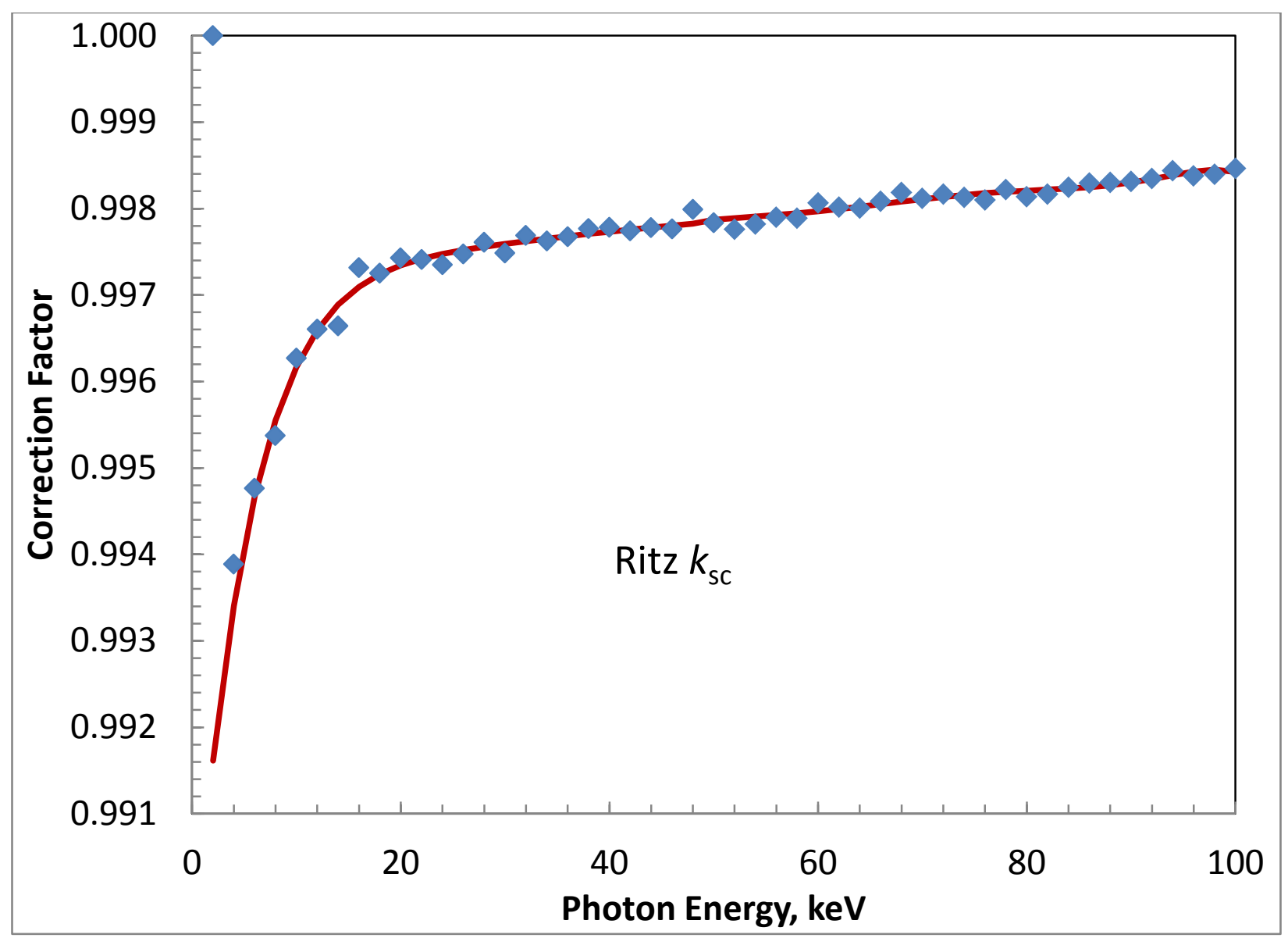

Figure 3. Comparison of original Burns data (points) for the Ritz free-air chamber with the results from the smoothed underlying data (curve).

b. Correction factor $k_{\mathrm{sc}}$. 


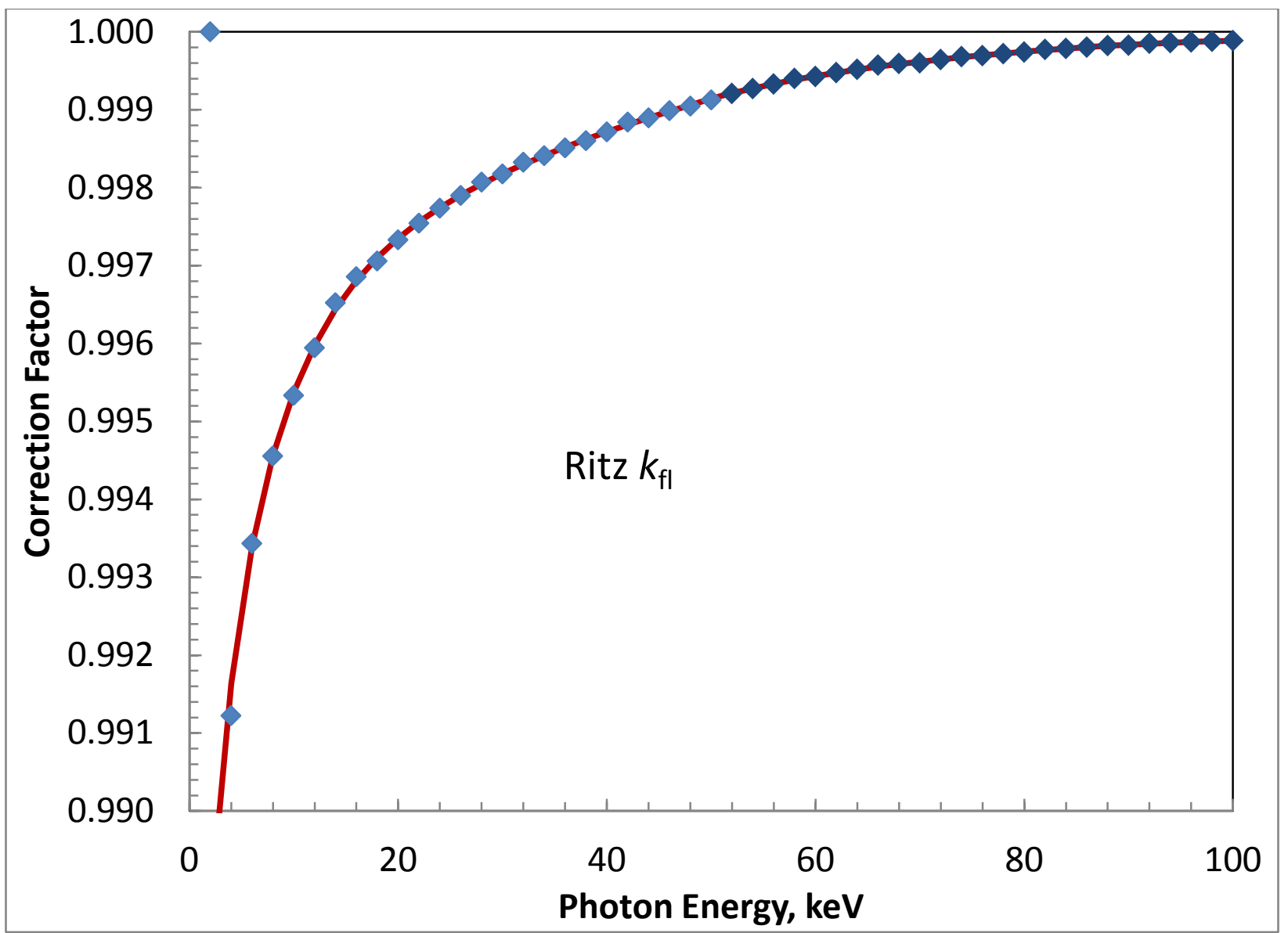

Figure 3. Comparison of original Burns data (points) for the Ritz free-air chamber with the results from the smoothed underlying data (curve).

c. Correction factor $k_{\mathrm{fl}}$. 


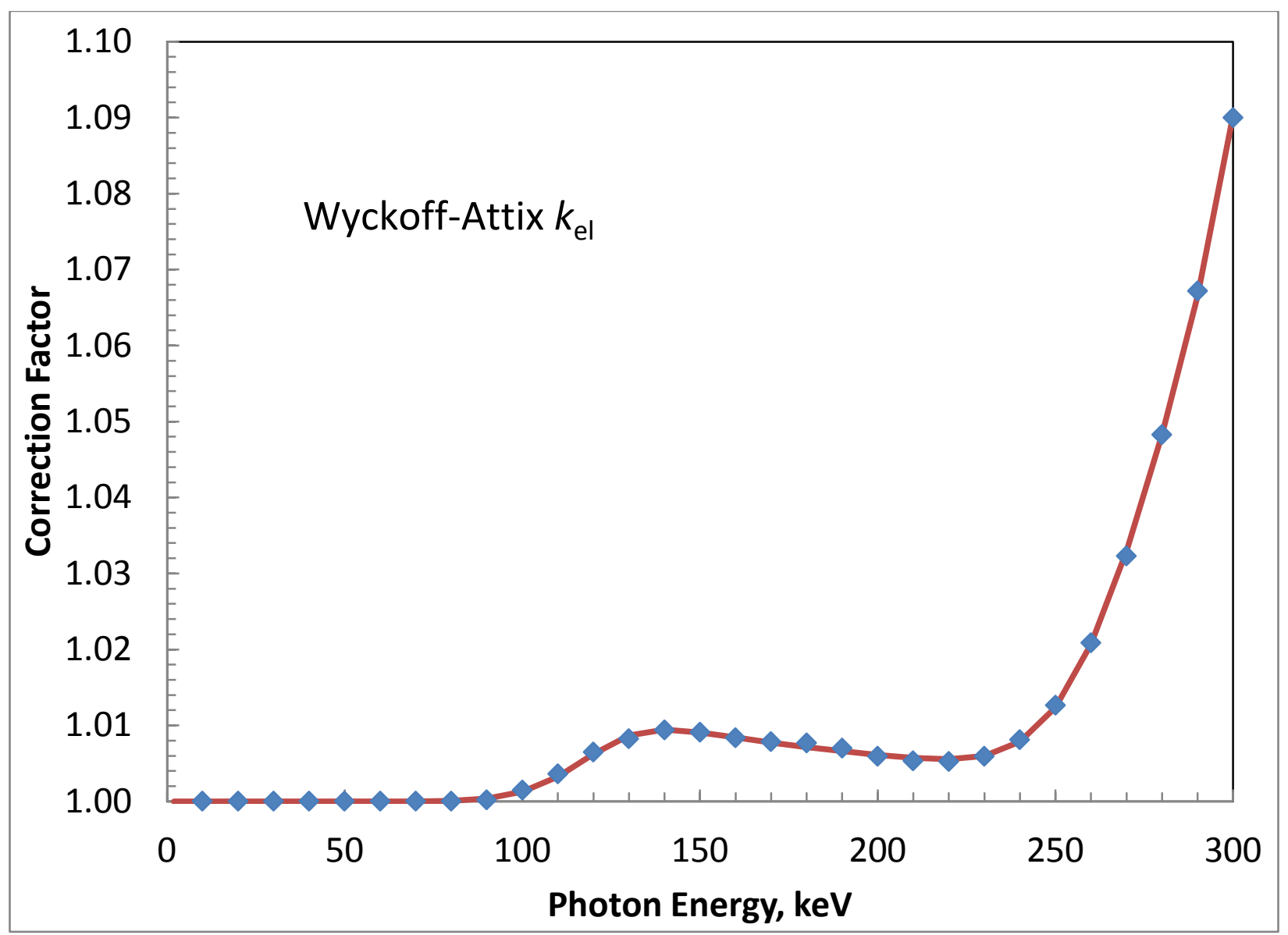

Figure 4. Comparison of original Burns data (points) for the Wyckoff-Attix free-air chamber with the results from the smoothed underlying data (curve).

a. Correction factor $k_{\mathrm{el}}$. 


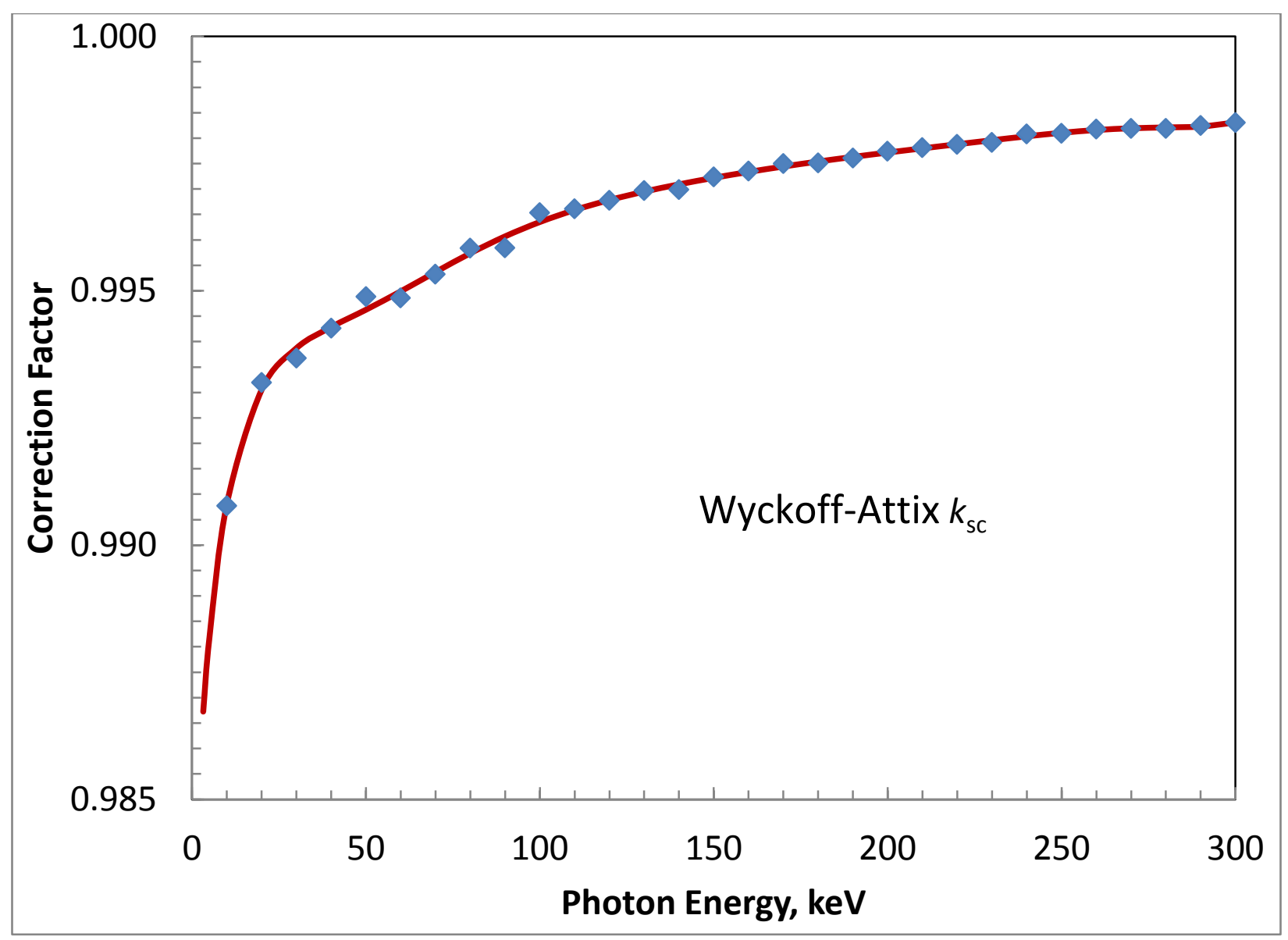

Figure 4. Comparison of original Burns data (points) for the Wyckoff-Attix free-air chamber with the results from the smoothed underlying data (curve).

b. Correction factor $k_{\mathrm{sc}}$. 


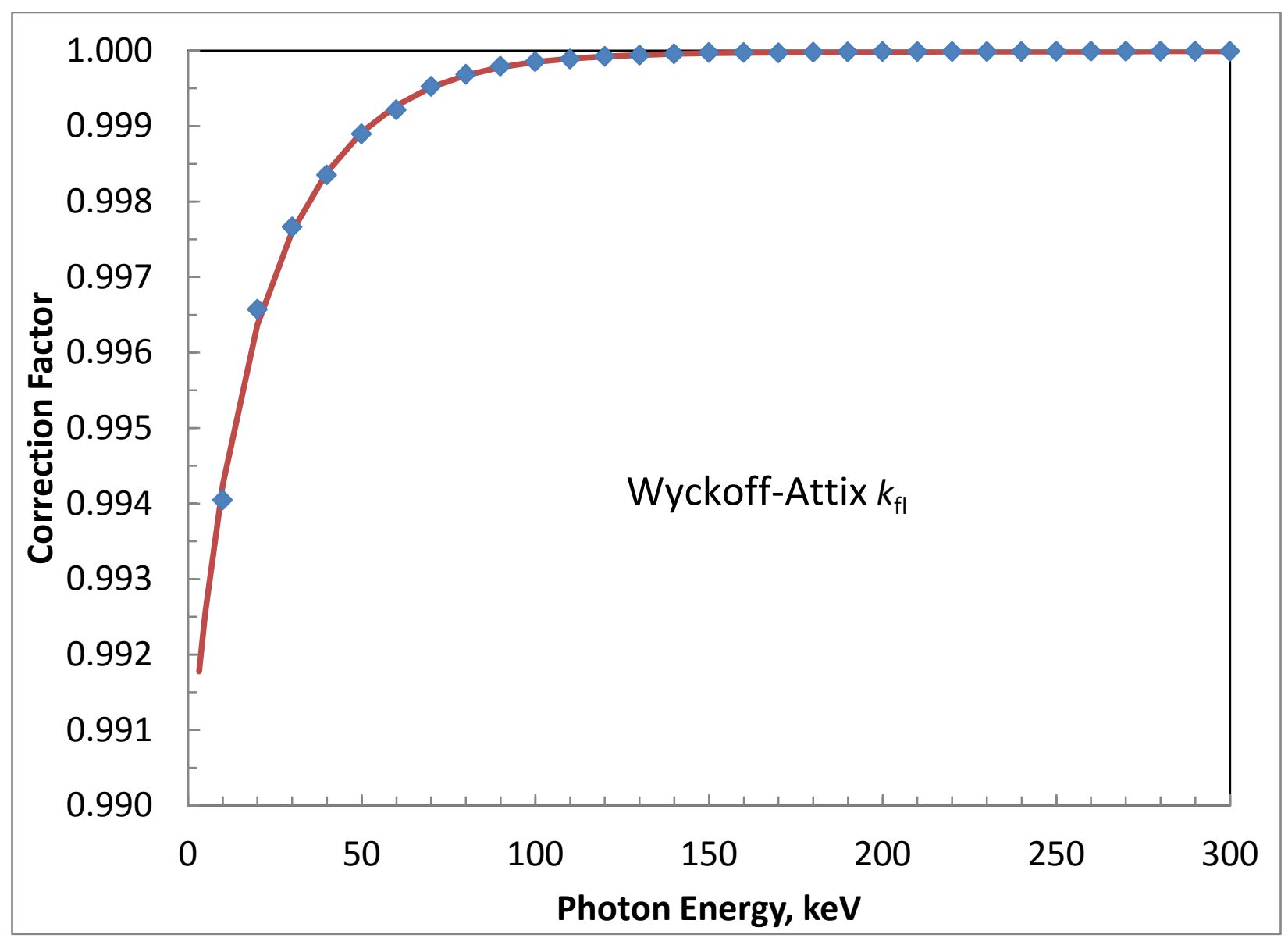

Figure 4. Comparison of original Burns data (points) for the Wyckoff-Attix free-air chamber with the results from the smoothed underlying data (curve).

c. Correction factor $k_{\mathrm{fl}}$. 